\title{
Quadrangulation through Morse-Parameterization Hybridization
}

\author{
XIANZHONG FANG, State Key Lab of CAD\&CG, Zhejiang University \\ HUJUN BAO*, State Key Lab of CAD\&CG, Zhejiang University \\ YIYING TONG, Michigan State University \\ MATHIEU DESBRUN, Caltech \\ JIN HUANG*, State Key Lab of CAD\&CG, Zhejiang University
}

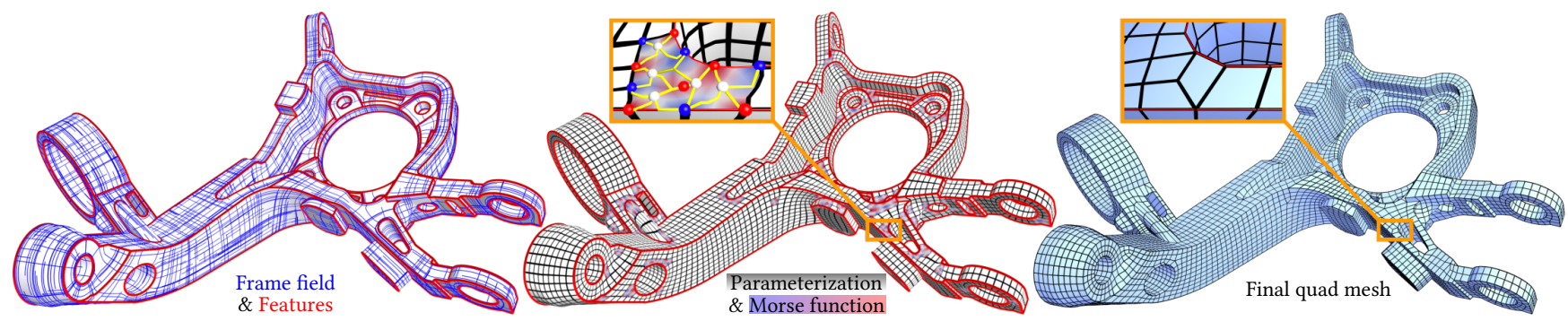

Fig. 1. Fast and robust hybrid quadrangulation via periodic vector field. We present an approach for efficiently producing frame- and feature-aligned quadrilateral meshes from input triangle meshes. By combining the efficiency of parameterization-based methods and the theoretical guarantees of Morsebased methods, our hybrid method robustly and efficiently extracts a non-degenerate quad mesh from a 4D periodic vector field computed from the input geometry and its frame field (here, a mechanical part model with complex feature lines).

\begin{abstract}
We introduce an approach to quadrilateral meshing of arbitrary triangulated surfaces that combines the theoretical guarantees of Morse-based approaches with the practical advantages of parameterization methods. We first construct, through an eigensolver followed by a few Gauss-Newton iterations, a periodic four-dimensional vector field that aligns with a user-provided frame field and/or a set of features over the input mesh. A field-aligned parameterization is then greedily computed along a spanning tree based on the Dirichlet energy of the optimal periodic vector field, from which quad elements are efficiently extracted over most of the surface. The few regions not yet covered by elements are then upsampled and the first component of the periodic vector field is used as a Morse function to extract the remaining quadrangles. This hybrid parameterization- and Morse-based quad meshing method is not only fast (the parameterization is greedily constructed, and the Morse function only needs to be upsampled in the few uncovered patches), but is guaranteed to provide a feature-aligned quad mesh with non-degenerate cells that closely matches the input frame field over an arbitrary surface. We show that our approach is much faster than Morse-based techniques since it does not require a densely tessellated input mesh, and is significantly more robust than parameterization-based techniques on models with complex features.
\end{abstract}

CCS Concepts: • Mathematics of computing $\rightarrow$ Mesh generation;

Additional Key Words and Phrases: Quadrangulation, periodic vector fields, frame fields, parameterization, Morse-Smale complex.

*Corresponding authors: $\{$ bao,hj\}@cad.zju.edu.cn.

Authors' addresses: X. Fang, H. Bao, J. Huang, State Key Lab of CAD\&CG, Zhejiang University, China; Y. Tong, Michigan State University, USA; M. Desbrun, Caltech, USA.

Permission to make digital or hard copies of all or part of this work for personal or classroom use is granted without fee provided that copies are not made or distributed for profit or commercial advantage and that copies bear this notice and the full citation on the first page. Copyrights for components of this work owned by others than ACM must be honored. Abstracting with credit is permitted. To copy otherwise, or republish to post on servers or to redistribute to lists, requires prior specific permission and/or a fee. Request permissions from permissions@acm.org.

(c) 2018 Association for Computing Machinery.

0730-0301/2018/8-ART92 $\$ 15.00$

https://doi.org/10.1145/3197517.3201354

\section{ACM Reference Format:}

Xianzhong Fang, Hujun Bao*, Yiying Tong, Mathieu Desbrun, and Jin Huang*. 2018. Quadrangulation through Morse-Parameterization Hybridization. ACM Trans. Graph. 37, 4, Article 92 (August 2018), 15 pages. https://doi.org/10. $1145 / 3197517.3201354$

\section{INTRODUCTION}

To this day, fully automated quadrangulation of surfaces with features and arbitrary guiding frame fields remains a challenge: producing a pure quad mesh with non-degenerate cells quickly and reliably while tightly adhering to a prescribed frame field indicating the desired edge directions and lengths is too often impossible without user interaction with current methods.

A first family of techniques (that we refer to as parameterizationbased quad meshing) relies on the singularity structure of the input frame field to derive a field-aligned parameterization, typically involving mixed integer optimization [Bommes et al. 2013a, 2009; Kälberer et al. 2007], from which a quad mesh can be extracted through isocontouring. While this general approach can be made computationally efficient, non-degeneracy of the parameterization is never guaranteed, in particular due to possible inconsistency between feature/boundary alignment, holonomy conditions and user constraints. A competing family of approaches (that we will denote as Morse-based quad meshing [Dong et al. 2006; Huang et al. 2008; Ling et al. 2014; Zhang et al. 2010]) exploits instead a key property of any Morse function: its induced Morse-Smale Complex (MSC) always corresponds to a valid quadrangulation [Edelsbrunner et al. 2003] - and so does its quasi-dual MSC [Dong et al. 2006]-as long as the function assumes different values for adjacent vertices, which can always be enforced through symbolic perturbation [Zomorodian 2009]. However, Morse-based methods require a very densely tessellated initial surface to be able to capture the highest frequencies of a Morse function (see Fig.2). Consequently, one can only 

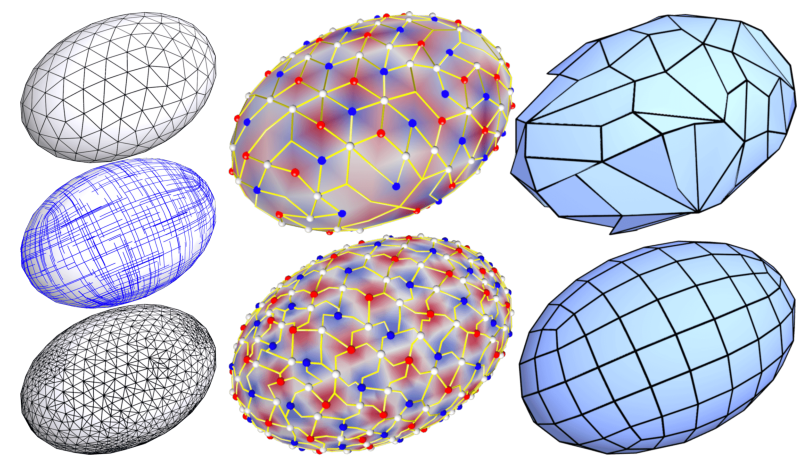

Fig. 2. Morse-Smale complex on coarse $\&$ dense meshes. A frame field from a coarse mesh (top left, 496 faces), can be interpolated on a finer mesh (bottom left, 3906 faces). A frame-aligned MSC (top middle) on the coarse mesh leads to a quad mesh with badly distorted elements (top right), while the quasi-MSC on a denser mesh of the same shape properly captures the input frame field (bottom middle), leading to a proper result (bottom right).

get proper quad meshes at a high computational cost: finding a finely-sampled Morse function with proper feature and frame field alignment requires a very large-scale optimization, making it less attractive for many practical applications.

In this paper, we introduce a hybridization of these two distinct approaches. First, a periodic 4D vector field is efficiently optimized over the (possibly coarse) initial mesh. Then a parameterization covering most of the input surface is greedily derived from this vector field, from which a valid (but incomplete) quad mesh is extracted. For the few remaining patches of the input surface not yet quadrangulated, we finally exploit the fact that, by construction, one of the components of our periodic $4 \mathrm{D}$ vector field is a Morse function: we can then refine these patches and upsample the Morse function locally to extract a Morse-derived quadrangulation that will exactly fill in the missing parts of the parameterization-derived quad mesh. As a result, we combine the efficiency of parameterization-based methods and the guarantees of Morse-based techniques to provide fast and robust frame- and feature-aligned quad meshing.

\subsection{Related work}

Due to the prevalence of quadrilateral meshes in computational methods and industrial design, the enduring issue of converting an arbitrary triangulated surface into a quad mesh that is aligned with surface features (and possibly with a user-provided frame field as well) has attracted a lot of interest over the years. The reader can find a comprehensive survey on existing techniques in [Bommes et al. 2013b]. In this section, we briefly review the approaches most related to our contribution.

Guiding frame fields. Cross-frame fields, or 4-RoSy vector fields, have been extensively explored starting with the work of Palacios and Zhang [2007]. Feature alignment and metric control of these fields are particularly relevant to quad meshing, as they allow a user to precisely define the orientation and length of edges of the expected resulting mesh [Jiang et al. 2015; Panozzo et al. 2014]. Various methods have been proposed to reduce or even remove the curl of the frame field to facilitate subsequent parameterization [Diamanti et al. 2015; Ray et al. 2006; Zhang et al. 2010]-see also [Vaxman et al. 2016] for a recent review.

Holonomy conditions. An issue rarely discussed (see [Lai et al. 2010], [Myles and Zorin 2013] and [Jiang et al. 2015] for notable exceptions) is that guidance frame fields are not always appropriate for describing a quad mesh layout: they need to verify some integrability conditions referred to as "holonomy conditions". More specifically, loops with the same homotopy type around a singularity of the frame field should have the same integer translation and multiple of $\pi / 2$ rotation in the parameter domain across a cut line from the singularity. In other words, the atlas derived from the frame field may not satisfy the holonomy constraints that a quad mesh requires. Since this constraint is never enforced in current frame field design techniques, one cannot guarantee that a proper pure quad mesh with edges well aligned with the frame field can actually be constructed from an arbitrary input without adding more singularities. Therefore, a frame field not satisfying these conditions brings difficulty to the quad meshing process regardless of the complexity of the actual shape of the surface.

Parameterization-based meshing. Most "parameterization-based" methods take a frame field as input, and construct an atlas according to the field singularities. Although this amounts mostly to a Poisson solve, the integer and folding-free constraints between charts bring additional complexity. While a simple greedy rounding [Bommes et al. 2009] and heuristic local stiffening can sometimes offer good parameterizations,[Bommes et al. 2013a] proposed a branch-andbound approach for increased robustness and efficiency. More recently, [Campen et al. 2015] introduced a method to generate integral global parameterizations by finding good quality quantizations. Interactive parameterizations were also obtained in [Ebke et al. 2016] by mapping a solution from a coarse mesh to a fine one. However, the success of these methods often hinges on the frame field satisfying the aforementioned holonomy conditions; otherwise the parameterization result is doomed to be degenerate, thus requiring user intervention. Myles and Zorin [2013] proposed to solve this issue by greedily adding singularities to reduce the parameterization distortion, but no orientation control was offered; in [Myles et al. 2014] the singularity structure is modified to enforce a local bijective and feature aligned parameterization, but integer constraints are not considered. Other parameterization-based methods have been proposed in the past. For instance, [Ray et al. 2006] proposed to solve for four periodic components over the mesh derived from the input cross field. However, the resulting quad-dominant mesh requires further splitting to make it a pure quad mesh, negatively impacting the alignment with the input guiding field. The fieldaligned mesh generation of Jakob et al. [2015] is highly efficient as it avoids performing a global optimization; an additional subdivision is, however, necessary to convert their quad-dominant result to a pure quad mesh. As a result, it leads to typically less accurate feature alignment than our method due to a lack of explicit feature constraints. An important limitation of all these methods is that only isotropic quad meshes can be generated in general: finding a parameterization that aligns well with an arbitrary anisotropic frame field has not been proven computationally robust so far. Also related are the works of [Kälberer et al. 2011] and [Knöppel et al. 2015] who 


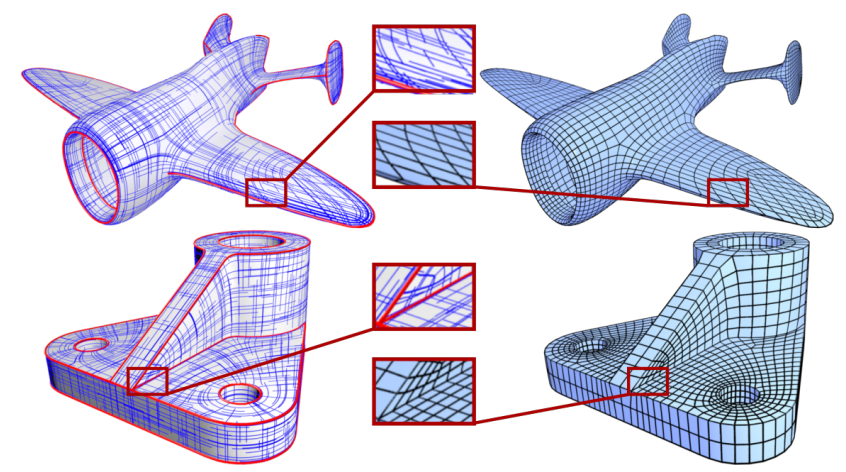

Fig. 3. Frame field control. Models with feature curves and frame fields controlling both local size, anisotropy and shearing of the elements.

proposed the construction of, respectively, stripe parameterizations for tubular surfaces with branched covering, and stripe patterns with line spacing and orientation control using periodic functions. However, these methods are limited to 2-fold symmetry, not 4-fold symmetry, and can thus not help with quad meshing directly.

Morse-based meshing. Another important, yet less explored tool for quadrangulation is the use of Morse functions. While the first Morse-based method of Dong et al. [2006] offered very limited control over the generated mesh, follow-up works were able to account for user constraints on direction, alignment and size [Huang et al. 2008; Ling et al. 2014; Zhang et al. 2010]. Extracting the final quad mesh is often achieved via its quasi-dual Morse-Smale complex: local maxima and minima of the Morse function are the vertices of the quads, while saddle points are at quad centers-or its primal variant. Because extraction relies expressly on the critical points being well separated, the Morse function has to be densely sampled: edge lengths of the input mesh have to be typically less than one fourth of the desired quad edge length. Alas, this implies that the input mesh has to be very dense, negatively affecting the efficiency of these Morse-based approaches since nonlinear optimization must be performed on meshes with very large vertex counts.

\subsection{Parameterization vs. Morse?}

Based on our review of prior work, we make several simple, yet important observations to motivate our approach:

- There has been no cross-pollination between parameterizationand Morse-based methods: both families rely on very distinct foundations, and have quite different properties and weaknesses;

- Using (composition of) trigonometric functions avoid the need for integer variables and their associated mixed-integer solvers;

- Frame-aligned parameterization often fails because of the presence of singularities and features: laying out a quad mesh along a trivial frame field over a smooth surface is very fast and simple;

- Extracting a mesh from a well-sampled Morse function is also rather simple (and guaranteed to be valid, with only pure quads), but getting this function to be aligned with features and a guiding frame field is often too computationally intensive due to inordinate requirements on mesh density;
- Yet, parameterizations and Morse functions are not inherently imcompatible: in fact, for any given Morse function, its dual MSC forms a quadrangulation, for which there exists a parameterization whose integer level sets coincide with the edges (see in-

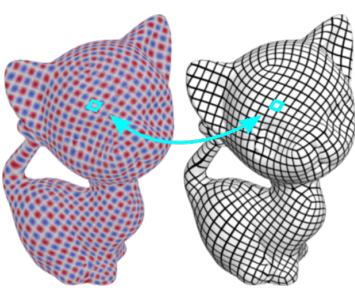
set). Consequently, a frame-aligned quadrangulation could be extracted on a surface through either a Morse function or an associated parameterization at various locations on the surface.

Based on these remarks, we propose what could be qualified as both a parameterization-based and a Morse-based approach: our contribution can be seen as bridging the gap between the two distinct families of methods by offering a hybrid technique that combines the efficiency of field-aligned parameterization and the theoretical guarantees of Morse functions.

\subsection{Overview}

In this paper, we present a fast, yet robust approach to construct frame- and feature-aligned quad meshes from arbitrary (non-degenerate) inputs. This is achieved by recognizing that a periodic multivalued field can be constructed to offer the means to derive both a parameterization and a compatible Morse function-thus providing simple and reliable tools to extract a pure quad mesh with nondegenerate cells (i.e., with a one-to-one mapping to the unit square) from any surface input with a frame field. More precisely, we introduce a number of contributions to reach our meshing goals.

Periodic multi-valued field. We make use of a 4D vector field similar to [Zhang et al. 2010] that we optimize to best capture the input frame field and mesh features. This key ingredient of our approach can be seen as an enhanced Morse function: one of its four components is guaranteed to form a Morse function; all of its components are also periodic, thus avoiding the need for integer variables and specific solvers that would come with them. Yet, the $4 \mathrm{D}$ field can be used to also derive a field-aligned parameterization compatible with the Morse function, thus offering two ways to extract the mesh from this $4 \mathrm{D}$ field.

Covariant derivative. We also leverage the trigonometric nature of the four components of the vector field to find a simple expression (through exponentiation) of a covariant derivative, which formally defines the notion of the "smoothest" $4 \mathrm{D}$ vector field constrained to align with the input frame field.

Eigenbased non-linear solve. The optimal smoothest $4 \mathrm{D}$ vector field is shown to be well approximated by an eigenvalue problem, which greatly accelerate (and improve the result of) the non-linear optimization always involved in quad meshing techniques.

Optimized singular structure. Unlike many previous works, the singularity structure of our resulting quad meshes is not necessarily the one from the input field, but the one of the optimized $4 \mathrm{D}$ vector field. Our approach is thus robust to arbitrary anisotropic input frame fields, even if they do not verify proper holonomy conditions.

Combined quad extraction. We then exploit the resulting periodic $4 \mathrm{D}$ vector field to extract a parameterization over most of the surface 


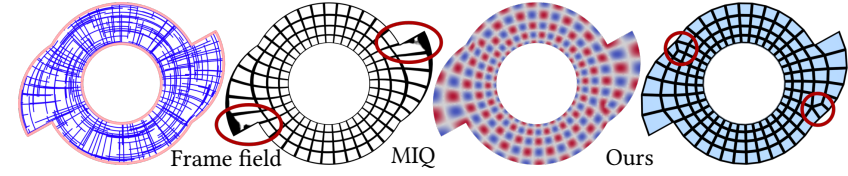

Fig. 4. Feature alignment. From a smooth, boundary-aligned input frame field (leftmost), a parameterization-based method [Bommes et al. 2009] creates degenerate elements (red marks, middle left); a frame-aligned Morse function (middle right) automatically adds four singularities (rightmost), so our hybrid method generates a valid quad mesh (rightmost).

through a greedy construction that inherently avoids the difficult treatment of singularities. The Morse component of the $4 \mathrm{D}$ field is then refined over the remaining, unparameterized patches only. A valid, pure quad mesh is then extracted using isocontouring of the parameterization and the quasi-dual Morse-Smale complex of the refined patches. Since both extractions derived from the same framealigned 4D vector field, they exactly match at their boundaries.

Finally, we demonstrate the robustness of our method on a large body of models: both surface inputs and quad outputs can be found in the Supplemental Material.

\section{FOUR-DIMENSIONAL PERIODIC VECTOR FIELD}

In this section, we introduce the main concepts and formulations underlying our approach to quad meshing.

\subsection{Background on parameterization-based meshing}

A common approach to quadrangulating a partitioned surface $\mathcal{S}$ is to find a mapping $\varphi$ from $\mathcal{S}$ to the plane,

$$
\varphi: \mathcal{S} \rightarrow \mathbb{R}^{2},
$$

whose gradients best align with a user-specified frame field $F$, expressed as a $2 \times 2$-matrix in an arbitrary local orthonormal frame per triangle. This is typically achieved by minimizing the alignment error within each chart while enforcing continuity across chart boundaries; i.e., one solves for the following minimization:

$$
\min _{\varphi} \int_{\mathcal{S}}\left\|\nabla \varphi-F^{-1}\right\|^{2} \quad \text { s.t. }\left\{\begin{array}{l}
\forall p \in C_{i} \cap C_{j}, \quad C_{i}, C_{j} \in C, \\
\varphi_{i}(p)=R^{k_{i j} \pi / 2} \varphi_{j}(p)+T_{i j} .
\end{array}\right.
$$

where $C$ denotes an atlas of charts on $\mathcal{S}$ with a transition between two charts $C_{i}$ and $C_{j}$ enforced along their shared border $C_{i} \cap C_{j}$. Continuity of the map is enforced up to a rotation $R^{k_{i j} \pi / 2}$ by an angle multiple of $\pi / 2$, and a translation $T_{i j}$ with integer components. However, such an approach may fail to guarantee a local bijective global parameterization for complex constraints: conflicts between feature alignment and continuity across charts often prevent the existence of a valid parameterization as illustrated in Fig. 4 (left).

In contrast, methods based on the MSC make use of a global Morse function, with no transitions needed across patch borders. Moreover, Morse-based methods are guaranteed to get bijective quadrangles (see for instance in Fig. 4, right), but only if a fine enough tessellation of the input surface is used. In practice, this requirement on mesh density poses a heavy computational burden that often scares away practitioners, despite its guaranteed robustness. Our approach combines these two existing strategies, using both a Morse function and a parameterization to offer robustness of the meshing process and a fine control over feature alignment. A periodic vector field is first optimized over the input mesh based on the input frame field and features. The extraction of a quad mesh is then achieved through isocurves of a greedily-constructed parameterization on most of the surface, while surface patches around singularities are treated through local refinement and Morse extraction.

\subsection{Background on Morse-based meshing}

A Morse function $g: \mathcal{S} \rightarrow \mathbb{R}$ over a surface $\mathcal{S}$ is a smooth function for which all critical points are non-degenerate; that is, the critical points $p \in \mathcal{S}$ of $g$ where the gradient $d g_{p}$ is zero must also have the determinant of the Hessian of $g$ to be non-zero, $|H(p)| \neq 0$. A Morse function can be defined over a partitioned surface through the composition $g=h \circ \varphi_{i}$ of a smooth function $h: \mathbb{R}^{2} \rightarrow \mathbb{R}$ acting on the parameterization $\varphi_{i}: \mathcal{S} \supset C_{i} \rightarrow \mathbb{R}^{2}$ of chart $C_{i}$, such that $g$ defines a function over the chart $C_{i}$ by composition. For the function $g$ to be continuous across the boundary between $C_{i}$ and $C_{j}, h$ has to satisfy $h \circ \varphi_{i}=h \circ \varphi_{j}$, hence:

$$
h\left(\varphi_{i}\right)=h\left(R^{k_{i j} \frac{\pi}{2}} \varphi_{j}+T_{i j}\right),
$$

given the integer transition conditions discussed above. A simple sufficient condition for such a function $h$ is thus to satisfy:

$$
h(\mathbf{u})=h(R \mathbf{u}), \quad \text { and } \quad h(\mathbf{u})=h(\mathbf{u}+T),
$$

for any rotation $R$ by an angle multiple of $\pi / 2$, any integer translation $T$, and any parameter coordinates $\mathbf{u}=(u, v)^{T}$.

Periodic Morse function. Zhang et al. [2010] used the periodic function $\cos \left(\omega_{x} x\right) \cos \left(\omega_{y} y\right)$ as a Morse function with local sizing and direction control, from which they generated a quadrangulation from its quasi-dual Morse-Smale complex where the quad cells are constructed using diagonals of the Morse-Smale cells [Dong et al. 2006]. If we treat $u=\omega_{x} x / \pi$ and $v=\omega_{y} y / \pi$ as the coordinates of a parameterization, the vertices of the resulting quad mesh correspond to integer grid points. This Morse function is thus a composition of the parameterization $(u, v)$ with the function:

$$
h(u, v)=\cos (\pi u) \cos (\pi v),
$$

which satisfies the conditions in Eq. (3) for translations $T=\left(T_{1}, T_{2}\right)^{T}$ such that the components are integers with the same parity, i.e.,

$$
T_{1} \pm T_{2} \in 2 \mathbb{Z} \text {. }
$$

Indeed, one can verify in this case that $2 h(u, v)=\cos (\pi(u+v))+$ $\cos (\pi(u-v))$ would be invariant under such translations. This is slightly more restrictive than the translation in Eq. (2), but necessary for Morse-based meshing as shown in Fig. 5. While this periodic function was key to enforcing feature alignment and extracting the resulting quadrangulation, their method suffered the typical
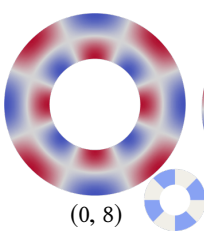

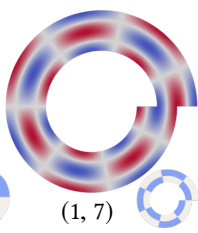

$(1,7)$

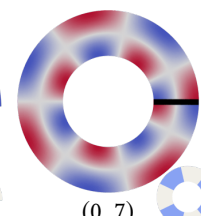

$(0,7)$

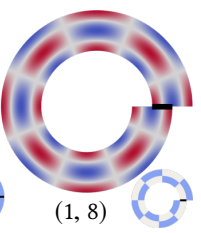

Fig. 5. Periodic Morse function. A parameterization of the annulus with a transition across a lateral cut satisfying $T_{1} \pm T_{2} \in 2 \mathbb{Z}$ (left two) can be transformed into a Morse function; without such a transition (right two), the resulting composition $h(u, v)$ will not be a periodic Morse function. 
drawbacks of Morse-based approaches. Instead, our work will use a four-dimensional periodic vector field, already introduced in [Zhang et al. 2010] but where only the first component was used for mesh extraction. We show that a global parameterization can be reliably recovered if the four components are exploited, and that this approach is robust even on coarse input triangle meshes, in marked contrast with existing Morse-based quad meshing techniques.

\subsection{Periodic four-dimensional vector field}

In our approach, we use a four-component periodic function $\psi$ :

$$
\psi(u, v)=(c c(u, v), s c(u, v), c s(u, v), s s(u, v))^{T},
$$

whose components are four periodic scalar functions defined as

$$
\left\{\begin{array}{l}
c c(u, v)=\cos (\pi u) \cos (\pi v) \\
s c(u, v)=\sin (\pi u) \cos (\pi v) \\
c s(u, v)=\cos (\pi u) \sin (\pi v) \\
s s(u, v)=\sin (\pi u) \sin (\pi v)
\end{array}\right.
$$

Further, we denote by $\Psi$ the four-dimensional vector field over the surface $\mathcal{S}$ defined by composing the periodic function $\psi$ with a parameterization $\varphi(p)=(u(p), v(p))^{T}$ :

$$
\Psi(p)=\psi \circ \varphi(p), \quad \forall p \in \mathcal{S} .
$$

Note that the first component $\left(\Psi^{0}=c c(u, v)\right)$ of this vector field is precisely the periodic Morse function $h$ discussed in Sec. 2.2. However, the other coordinates do not form Morse functions as they are not invariant under $\pi / 2$ rotation of the parameterization: denoting the counterclockwise rotation of $\mathbb{R}^{2}$ by $\pi / 2$ as $J$, one has

$$
\psi(J \mathbf{u})=\hat{J} \psi(\mathbf{u})
$$

instead, where $\hat{J}$ is a matrix performing a permutation of the components up to a sign as easily verified via trigonometric identities:

$$
\hat{J}=\left(\begin{array}{cccc}
1 & 0 & 0 & 0 \\
0 & 0 & -1 & 0 \\
0 & 1 & 0 & 0 \\
0 & 0 & 0 & -1
\end{array}\right) .
$$

This implies that the vector field $\Psi$ satisfies the following property at the boundary between two charts $C_{i}$ and $C_{j}$ :

$$
\Psi(p)=\psi\left(\varphi_{i}(p)\right)=\psi\left(J^{k_{i j}} \varphi_{j}(p)+T_{i j}\right)=\hat{J}^{k_{i j}} \psi\left(\varphi_{j}(p)\right),
$$

where the translations $T_{i j}$ between charts are naturally canceled by our choice of the four periodic components of $\psi$, and where we denoted the transition rotation between charts $i$ and $j$ by an angle of $k_{i j} \pi / 2$ as $J^{k_{i j}}$. Finally, we note that our choice of the fourcomponent periodic function trivially implies the following properties for the vector field $\Psi$ due to the use of trigonometric functions:

$$
\|\Psi\|_{2}=1 \quad \text { and } \quad \Psi^{0} \Psi^{3}=\Psi^{1} \Psi^{2} .
$$

Our approach will look for a smooth 4 D vector field $\Psi$ satisfying these simple relationships between all four components, so as to define a quad mesh with non-degenerate cells (i.e., cells with a one-to-one map to the square).

\subsection{Differential property of $4 \mathrm{D}$ vector field}

The differential of the periodic function $\psi(u, v)$ is easily found, using the derivatives of sin and cos, to be

$$
d \psi=\pi W(d u, d v) \psi,
$$

where $W$ is the matrix-valued function

$$
W(d u, d v)=\left[\begin{array}{cccc}
0 & -d u & -d v & 0 \\
d u & 0 & 0 & -d v \\
d v & 0 & 0 & -d u \\
0 & d v & d u & 0
\end{array}\right]
$$

This property implies that a vector field $\Psi$ based on $\psi$ and a parameterization $\varphi(p)=(u(p), v(p))$ has to satisfy (by chain rule):

$$
\frac{\partial \Psi}{\partial p}-\pi W\left(\frac{\partial u}{\partial p}, \frac{\partial v}{\partial p}\right) \Psi=0 .
$$

Since we wish to construct a vector field $\Psi$ before deducing a parameterization $\varphi$ from it, we instead exploit the input guidance frame field $F$, with which we want $d \varphi$ to be aligned: we form a connection (or covariant derivative [Crane et al. 2010]) $\hat{\nabla} \Psi$ defined on the $4 \mathrm{D}$ vector bundle $\mathcal{S} \times \mathbb{R}^{4}$, which, for any tangent vector $\mathbf{v}$, is defined as:

$$
\hat{\nabla}_{\mathbf{v}} \Psi=\frac{\partial \Psi}{\partial p} \mathbf{v}-\pi W\left(F^{-1} \mathbf{v}\right) \Psi .
$$

Note that we have effectively removed the explicit use of the parameterization: this differential expression should hold if the vector field $\Psi$ were to derive from an $F$-aligned parameterization.

\subsection{Feature and boundary alignment}

Feature and boundary alignment are easily incorporated as constraints on $\Psi$ as proposed in [Zhang et al. 2010]. Indeed,

- for any point $p$ along a feature or a boundary, one must have $u(p) \in \mathbb{Z}$ or $v(p) \in \mathbb{Z}$. This is achieved by enforcing:

$$
\Psi^{3}(p)=s s(u(p), v(p))=0 ;
$$

- for any corner point $p$, i.e., a sharp corner along a feature or boundary, we need $u(p) \in \mathbb{Z}$ and $v(p) \in \mathbb{Z}$. Thus we simply force the components $s c, c s, s s$ of $\Psi$ to be zero:

$$
\Psi^{1}(p)=\Psi^{2}(p)=\Psi^{3}(p)=0
$$

\subsection{Optimal periodic vector field}

In order to obtain the simplest layout that satisfies all feature and boundary alignments, we search for the smoothest $\Psi$ for a given guidance frame field $F$ by simply minimizing the Dirichlet energy

$$
\mathcal{E}(\Psi)=\int_{\mathcal{S}}\|\hat{\nabla} \Psi\|^{2} \quad \text { s.t. }\left\{\begin{array}{l}
\|\Psi\|_{2}=1, \Psi^{0} \Psi^{3}=\Psi^{1} \Psi^{2}, \\
\text { and all feature constraints. }
\end{array}\right.
$$

We will show that this optimization of a $4 \mathrm{D}$ vector field is actually simple to perform reliably by exploiting the fact that, once the first two constraints are omitted, it corresponds to a simple quadratic functional minimization.

\section{PERIODIC VECTOR FIELD GENERATION}

We now explain how the geometric approach we described above is easily implemented and solved numerically.

\subsection{Discrete setup}

Given a piecewise-linear approximation $\mathcal{M}$ of a surface $\mathcal{S}$, with vertices $\mathcal{V}$ and triangles $\mathcal{T}$, we store the user-prescribed input frame field as one frame $F_{t}$ per triangle $t \in \mathcal{T}$ [de Goes et al. 2015]. We discretize our periodic $4 \mathrm{D}$ vector field at vertices as a fourcomponent vector $\Psi_{p}$ on each vertex $p \in \mathcal{V}$, and the vector stacking all the values $\Psi_{p}$ is denoted as $\Psi$. Since the expression of $\Psi_{p}$ depends 
on the local frame, we have to define one frame per vertex as well. Without loss of generality, we pick the vertex frame $F_{p}$ to be the frame $F_{t_{0}}$, where $t_{0}$ is one triangle in the one-ring of $p$.

\subsection{Discretization of smoothness energy}

Half-edge based discretization. Based on our discrete setup, we discretize the integral of $\|\hat{\nabla} \Psi\|^{2}$ by summing up one term per half edge, or equivalently, two terms per edge $(p, q) \in \mathcal{E}$ as we now detail. For a half edge $\mathbf{e}_{p q}$ from vertex $p$ to vertex $q$ in triangle $t$, the parallel-transport of $\Psi_{p}$ along $\mathbf{e}_{p q}$ in the connection $\hat{\nabla}$ defined by $F_{t}$ is $\exp \left(\pi W\left(F_{t}^{-1} \mathbf{e}_{p q}\right)\right) \Psi_{p}$ [Liu et al. 2016] since the continuous field $\Psi$ obeys the differential property given in Eq. (11). The edge contribution to the discrete version of the smoothness energy $\mathcal{E}(\Psi)=$ $\int_{\mathcal{S}}\|\hat{\nabla} \Psi\|^{2}$ is thus:

$$
\mathcal{E}_{p q}=\frac{|t|}{2\left|\mathbf{e}_{p q}\right|^{2}}\left\|\Psi_{q, t}-\exp \left(\pi W\left(F_{t}^{-1} \mathbf{e}_{p q}\right)\right) \Psi_{p, t}\right\|^{2},
$$

where $|t|$ is the area of triangle $t$, and $\Psi_{p, t}$ is the expression of $\Psi_{p}$ in the frame of $t$, the latter being evaluated as $\Psi_{p, t}=\hat{J}^{k_{p t}} \Psi_{p}$ where $k_{p t}$ encodes the integer multiple of $\pi / 2$ rotation between point $p$ and triangle $t$. (We will provide in the next paragraph a frame alignment value for $k_{p t}$ based on the input frame field). Note that the matrix exponential can be evaluated in closed form, since:

$$
\exp (\pi W)=\left[\begin{array}{cccc}
c c(d u, d v) & -s c(d u, d v) & -c s(d u, d v) & s s(d u, d v) \\
s c(d u, d v) & c c(d u, d v) & -s s(d u, d v) & -c s(d u, d v) \\
c s(d u, d v) & -s s(d u, d v) & c c(d u, d v) & -s c(d u, d v) \\
s s(d u, d v) & c s(d u, d v) & s c(d u, d v) & c c(d u, d v)
\end{array}\right]
$$

Frame alignment calculation. The input frame field $F$, given as a frame for each face of the input triangle mesh, can be a cross field or an arbitrary, non-orthogonal frame field [Jiang et al. 2015; Panozzo et al. 2014]. We compute the alignment angles in Eq. (15) based on the frames of the two faces across a common edge: for two adjacent triangles $s, t \in \mathcal{T}$, the best alignment angle $k_{s t} \pi / 2$ between frame $F_{s}$ and frame $F_{t}$ is computed through

$$
k_{s t}=\underset{k}{\operatorname{argmin}}\left\|F_{s} J^{4-k}-F_{t}\right\|^{2}, k \in\{0,1,2,3\},
$$

where $F_{s}$ and $F_{t}$ are first aligned through the Levi-Civita connection across the common edge [de Goes et al. 2015].

For the local frame needed to express $\Psi$ at each vertex $p$, we simply use the frame of one of the adjacent faces $t_{0}$. The best local alignment from $p$ to that face then becomes $k_{p t_{0}}=0$. Assuming the faces incident to $p$ are $\left(t_{0}, t_{1}, \ldots, t_{m}\right)$ in counterclockwise order, the best alignment for the other faces are simply given as

$$
k_{p t_{j}}=\left(\sum_{i=0}^{j-1} k_{t_{i} t_{i+1}}\right) \bmod 4 .
$$

Constrained optimization. Summing up all the half edge contributions, we can now formulate the optimization of the discretized smoothness energy $\mathcal{E}(\Psi)=\sum$ edge $(p, q) \mathcal{E}_{p q}$ (quadratic in $\Psi$ ) as

$$
\min _{\Psi} \mathcal{E}(\Psi) \text { s.t. }\left\{\begin{aligned}
\left\|\Psi_{p}\right\|^{2} & =1 \text { and } \Psi_{p}^{0} \Psi_{p}^{3}=\Psi_{p}^{1} \Psi_{p}^{2}, & & p \in \mathcal{V} \\
\Psi_{p}^{3} & =0, & & p \in \mathcal{V}_{b} \\
\Psi_{p}^{1} & =\Psi_{p}^{2}=0, & & p \in \mathcal{V}_{c}
\end{aligned}\right.
$$

where $\mathcal{V}_{b}$ is the set of vertices on boundaries and feature curves, and $\mathcal{V}_{c}$ is the set of corner vertices, i.e., vertices that belong to multiple feature/boundary edges.
Further improvements. When the input mesh is too coarse compared to the metric $g=\left(F F^{\top}\right)^{-1}$ defined by the input frame field, setting constraint on the discrete point set $\mathcal{V}_{b}$

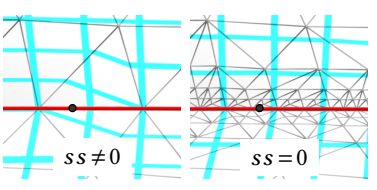
may fail to enforce the condition along feature and boundary curves in Eq. (12). In this case, setting $s s(p)=0$ on vertices may no longer ensure that $s s$ is zero along the whole feature or boundary edges (see inset, left; feature in red, isolines in blue). We address this situation by performing mid-edge subdivision of edges connecting boundary and feature vertices until the length of each edge connecting boundary and feature vertices is below a threshold $\varepsilon_{b}$ in the metric $g$ (see inset, right). The value $\varepsilon_{b}=0.5$ is fail-safe, since skipping from one isocurve to another within an edge can only occur if the edge is longer than the threshold in the local metric $g$.

Moreover, feature and boundary alignment can be further improved through a simple weighting, making both alignment more exact and reducing the number of quadrangles with bad quality nearby: we just increase the weight to better control frame alignment energy near features and boundaries; that is, we define:

$$
\begin{gathered}
\mathcal{E}(\Psi)=\frac{1}{W_{\text {sum }}} \sum_{p q \notin \mathcal{N}_{\mathrm{b}-\mathrm{f}}} \mathcal{E}_{p q}+\frac{\mu}{W_{\text {sum }}} \sum_{p q \in \mathcal{N}_{\mathrm{b}-\mathrm{f}}} \mathcal{E}_{p q} \\
\text { for } W_{\text {sum }}=\sum_{p q \notin \mathcal{N}_{\mathrm{b}-\mathrm{f}}} \frac{|t|}{2\left|\mathbf{e}_{p q}\right|^{2}}+\mu \sum_{p q \in \mathcal{N}_{\mathrm{b}-\mathrm{f}}} \frac{|t|}{2\left|\mathbf{e}_{p q}\right|^{2}}
\end{gathered}
$$

instead, where $\mathcal{N}_{\mathrm{b}-\mathrm{f}}$ is the set of edges connecting boundary or feature vertices, and $\mu \geq 1$ is the alignment enforcement weight ( $\mu=20$ in our implementation).

Finally, the corner constraint set $\mathcal{V}_{c}$ is automatically constructed at the intersection of several feature and/or boundary edges. Additionally, if the angle $\theta$ between two adjacent boundary edges is below a threshold $\varepsilon_{\theta}$ (we chose $100^{\circ}$ ), we treat it as an intersection, and create a corner constraint there. For each corner, we use Eq. (13) to ensure integer grid values for the parameterization.

\subsection{Eigensolver}

As noted in Sec. 2.6, the discretized Dirichlet energy $\mathcal{E}$ is a quadratic form in $\Psi$. We can exploit this property to obtain a very good guess of the optimal $4 \mathrm{D}$ field $\Psi$ : by removing the vertex-wise non-linear conditions $\left\|\Psi_{p}\right\|_{2}=1$ and $\Psi_{p}^{0} \Psi_{p}^{3}=\Psi_{p}^{1} \Psi_{p}^{2}$ from the above minimization, we turn the optimization into an eigenproblem by adding the constraint $\|\Psi\|_{2}=1$ to avoid the degenerate solution $\Psi=\mathbf{0}$. In other words, we compute a first approximation of the optimal field $\Psi$ by solving the following eigenvalue problem:

$$
\mathrm{H} \Psi=\lambda_{\min } \Psi,
$$

where $\mathbf{H}$ is the quadratic form approximating the Dirichlet energy. Indeed, the vertex constraints on features, boundaries and corners can be taken care of by removal of the corresponding variables from $\Psi$. We thus begin by solving for the eigenvector $\Psi$ associated with the smallest eigenvalue of $\mathbf{H}$ as a good estimate of the smoothest vector field $\Psi$. From this solution, we project the vector at each vertex with $\Psi_{p} \leftarrow \psi \circ \varphi^{*}(p)$ (see Eq. (19)) to trivially enforce the nonlinear constraints that we had omitted. We then perform a classical penalty-based nonlinear optimization through the Gauss-Newton 
method to solve for the original constrained minimization:

$$
\begin{array}{ll}
\min _{\Psi} & \mathcal{E}(\Psi)+\frac{w_{r}}{|\mathcal{V}|} \sum_{p \in \mathcal{V}}\left(\left(\left\|\Psi_{p}\right\|_{2}^{2}-1\right)^{2}+\left\|\Psi_{p}^{0} \Psi_{p}^{3}-\Psi_{p}^{1} \Psi_{p}^{2}\right\|_{2}^{2}\right) \\
\text { s.t. } & \Psi_{p}^{3}=0, \quad p \in \mathcal{V}_{b} ; \quad \Psi_{p}^{1}=\Psi_{p}^{2}=0, \quad p \in \mathcal{V}_{c} .
\end{array}
$$

where the weight $w_{r}$ allows for the enforcement of the nonlinear constraints $\left(w_{r}=1\right.$ in our implementation, and we assume convergence when gradient magnitude is below $1 e-3$ ). We eliminate the constrained variables from the target function, replacing them by their values directly.

We will show in Sec. 5 that this two-step procedure is very efficient as the eigenvalue problem provides a close estimate of the final solution, making the non-linear solve much more robust against local minima (see Figs. 15 and 16). This is a particularly key feature of our seemingly-redundant $4 \mathrm{D}$ vector field: it allows us to turn the highly non-linear problem of frame alignment into a constrained minimization well approximated by an eigenvalue problem.

\section{FROM PERIODIC FIELD TO QUADRANGULATION}

From the periodic vector field resulting from our constrained energy minimization, a global parameterization needs to be extracted. We offer a fast two-stage approach which greedily constructs a parameterization in regions of low Dirichlet energy first, then exploits the Morse function $\Psi^{0}$ in regions where the greedy construction failed to provide a satisfactory parameterization to quadrangulate. More specifically, after the greedy parameterization stage, we extract quadrangles centered at points with half-integer parameter values. The union of these quadrangles is denoted $Q_{R}$, the complement of which is defined as the singular region $\mathcal{M}_{S}$. In the Morse-based stage, we refine $\Psi$ in $\mathcal{M}_{S}$ by solving the constrained optimization on a locally refined mesh to obtain a high-quality MSC, with the original $\Psi$ fixed on the common boundary $Q_{R} \cap \mathcal{M}_{S}$ to ensure flawless compatibility between the two regions, see Fig. 6 . This technique allows us to extract a pure quad mesh efficiently as we now detail.

\subsection{Overview}

Once a periodic field $\Psi$ is known, finding a corresponding parameterization $\varphi$ is no easy task: there is an infinite number of solutions differing by integer offsets. Indeed, suppose we know a parameterization $\varphi^{*}$ which matches $\Psi$, then parameterizations of the form

$$
\varphi(p)=\varphi^{*}(p)+(a, b)^{T}, \quad a \pm b \in 2 \mathbb{Z}
$$

are also valid due to the periodicity of $\psi$.

From all possible parameterizations, we need to construct one that is best aligned with the input frame field; that is, one for which $\int_{\mathcal{M}}\left\|\nabla \varphi-F^{-1}\right\|^{2}$ is small. However, optimizing frame alignment through integer programming while forcing the parameterization to be seamless is computationally intractable in practice. Directly exploiting the fact that the first component of $\Psi$ is a Morse function will also fail for coarse input meshes. We thus propose to proceed instead in two steps:

- First, a local parameterization $\varphi^{*}(p)=\left(u^{*}(p), v^{*}(p)\right)^{T}$ is assigned to each vertex $p$ based on the value of $\Psi_{p}$. From these local parameter values, we greedily construct a valid "spanning parameterization" $\varphi$ that covers most of the input surface through an efficient Dirichlet energy guided mesh traversal. Here we leverage the fact that a low local Dirichlet energy of the optimal periodic vector field implies smoothness, hence easy construction of a parameterization. From this spanning parameterization, we can extract most of the quad mesh;

- We then exploit the Morse function $\Psi^{0}$ to extract the missing quadrangles using the quasi-dual Morse-Smale complex found after refinement of the input mesh in the remaining regions.

We now review these two steps and discuss why they guarantee the fast extraction of a seamless, pure quad mesh.

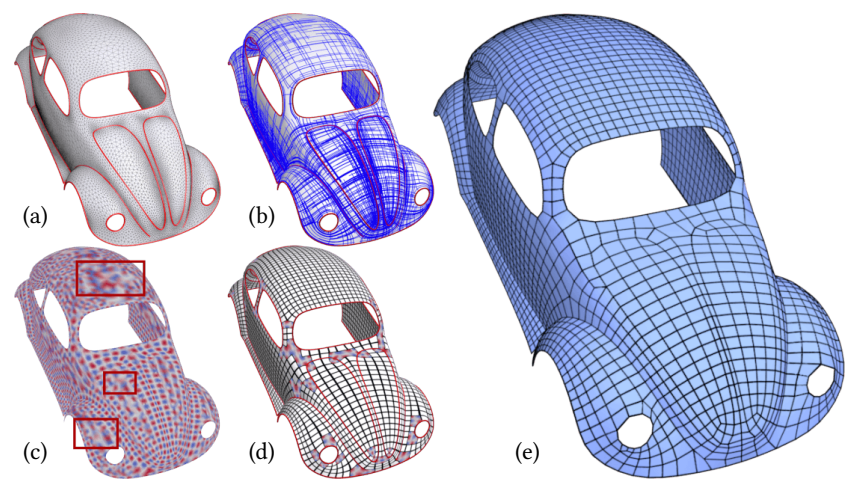

Fig. 6. Hybrid Quadrangulation. (a) The input triangle mesh of the beetle car with its features (red curves), and (b) an input frame field (blue strokes). (c) The $c c$ component of $\Psi$; red boxes highlight regions where Morse-based methods cannot extract an appropriate complex without prior mesh refinement. (d) Patches from the singular region (showing $c c$ component) within the regular region (quad texture). (e) Our result showing perfect fit between the two extracted quadrangulations, as expected.

\subsection{Spanning parameterization}

The spanning parameterization, which will cover most of the surface except for a small number of patches, is found by growing a parameterization greedily along a spanning tree based on the Dirichlet energy of the optimal periodic vector field.

Assignment of local parameters. From the optimized periodic vector field, we assign at each vertex $p$ a pair of compatible parameters $\left(u^{*}(p), v^{*}(p)\right)^{T}=\varphi_{p}^{*}$ through:

$$
\varphi^{*}(p)=\frac{1}{2 \pi}\left(\begin{array}{l}
\operatorname{atan} 2\left(\Psi_{p}^{1}+\Psi_{p}^{2}, \Psi_{p}^{0}-\Psi_{p}^{3}\right)-\operatorname{atan} 2\left(\Psi_{p}^{1}-\Psi_{p}^{2}, \Psi_{p}^{0}+\Psi_{p}^{3}\right) \\
\operatorname{atan} 2\left(\Psi_{p}^{1}+\Psi_{p}^{2}, \Psi_{p}^{0}-\Psi_{p}^{3}\right)+\operatorname{atan} 2\left(\Psi_{p}^{1}-\Psi_{p}^{2}, \Psi_{p}^{0}+\Psi_{p}^{3}\right)
\end{array}\right),
$$

where atan 2 is the function that returns the angle $\in(-\pi, \pi]$ given its sine and its cosine as arguments. In order to satisfy the boundary and feature conditions exactly, we further snap some of the local coordinates of corner, boundary, or feature vertices onto their nearest integer: for corner vertices $p \in \mathcal{V}_{c}$, we snap both coordinates $u^{*}(p) \leftarrow \operatorname{round}\left[u^{*}(p)\right]$ and $v^{*}(p) \leftarrow \operatorname{round}\left[v^{*}(p)\right]$; similarly, for feature/boundary vertices $p \in \mathcal{V}_{b}$, we set $u^{*}(p) \leftarrow \operatorname{round}\left[u^{*}(p)\right]$ or $v^{*}(p) \leftarrow \operatorname{round}\left[v^{*}(p)\right]$ depending on whether the feature/boundary edge passing through $p$ is a $v$ - or $u$-isoline in the local frame at $p$. Note that the position field in [Jakob et al. 2015] resembles our local parametric coordinates $\varphi^{*}(p)$. However, their expression cannot handle explicit constraints for feature edges (neither crease nor non-crease ones), in contrast to our method. 
Construction of spanning tree. Given local parametric coordinates $\varphi^{*}(p)$, we wish to find a "spanning" parameterization $\varphi$ by advancing along a spanning tree of the dual graph of the mesh. To find a good spanning tree through which the parameterization can easily be computed, we drive the construction of the spanning tree based on the value of the smoothness energy $\mathcal{E}(\Psi)$. More precisely, we define the Dirichlet energy for each triangle $t$ as the sum of the energy values of its edges defined in Eq. (15),

$$
\mathcal{E}_{t}=\sum_{(p, q) \subset t \in \mathcal{T}}\left(\mathcal{E}_{p q}+\mathcal{E}_{q p}\right) .
$$

The root of the spanning tree is then set on the face $t_{\min }$ with the minimum triangle energy value. The triangle $t_{\mathrm{min}}$ and its adjacent neighbors are then pushed onto a heap of unvisited triangles, with the triangle energy value as the key. We then repeat the following operations until the heap is empty: remove the triangle with the minimum key from the heap, add the dual edge to the adjacent visited node to the tree, and push its adjacent triangles onto the heap. This procedure forms a spanning tree that cuts the input mesh into a topological disk $\mathcal{M}_{c}$.

Greedy construction of parameterization. We are now ready to construct the spanning parameterization. We treat each triangle as its own chart, such that a vertex $p$ is assigned a different parameter value per adjacent triangle. We will denote $\varphi_{t}(p)$ the coordinates of $p$ within the chart formed by triangle $t$.

With the spanning tree and its implied topological disk, we can now remove the constraints of transition between triangle charts by simply setting the transition for all tree edges to zero rotation and zero translation. We then traverse the spanning tree we just constructed, and layout triangle-by-triangle the spanning parameterization that minimizes frame alignment distortion with respect to the frame field $F$. We begin by setting $\varphi_{t_{\min }}(p)=\varphi^{*}(p)$ for one of the vertices of $t_{\min }$. The counterclockwise neighbor $q$ of $p$ in $t_{\min }$ is then given the parametric coordinates $\varphi_{t_{\min }}(q)=\varphi^{*}(q)+(a, b)^{T}$ with $a \pm b \in 2 \mathbb{Z}$ such that the integrated gradient $\varphi_{t_{\min }}(q)-\varphi_{t_{\min }}(p)$ best matches the desired parametric integrated gradient $d \varphi$ along the edge $\mathbf{e}_{p q}$ in $F$. As we traverse the tree (i.e., as we cross edge $(p, q)$ to go from triangle $s=(m, p, q)$ to $t=(p, r, q))$, we begin by copying the parameter values of the two vertices of the edge such that $\varphi_{t}(p)=\varphi_{s}(p)$ and $\varphi_{t}(q)=\varphi_{s}(q)$-hence imposing a chart transition with no rotation and no translation. We then need to find the parameter coordinates $\varphi_{t}(r)$ for the unvisited vertex $r$ which minimizes frame alignment distortion and find it by solving:

$$
\begin{aligned}
\min _{\varphi_{t}(r)} & \left\|\varphi_{t}(r)-\varphi_{t}(p)-d \varphi_{p r}\right\|^{2}+\left\|\varphi_{t}(r)-\varphi_{t}(q)-d \varphi_{q r}\right\|^{2} \\
\text { s.t. } & \left\{\begin{array}{l}
\varphi_{t}(r)=R^{\left(k_{r t}+k_{t}\right) \frac{\pi}{2}} \varphi^{*}(r)+\left(T_{t, r}^{0}, T_{t, r}^{1}\right)^{T}, T_{t, r}^{0} \pm T_{t, r}^{1} \in 2 \mathbb{Z}, \\
\operatorname{det}\left(\nabla \varphi_{t}\right)>0 .
\end{array}\right.
\end{aligned}
$$

Here, $k_{t}$ denotes the rotation determined by the spanning tree through $k_{t}=k_{t s}+k_{s}$ for $s$ being the parent node of $t$, and we set the root rotation $k_{t_{\min }}$ to 0 . This minimization is trivially solved by first computing the optimal parameter values $\varphi_{r}$ without the integer constraints, then testing the nine nearby integer grid points to find the best valid match. Once $\varphi_{t}(r)$ is found, we move on to the next tree edge. By the end of the tree traversal, we have a spanning parameterization with low frame alignment distortion almost everywhere, see Fig. 8.
Robustness against floating point operations. In order to avoid floating point issues during the extraction of quadrangles from the parameterization, we add one last rounding of the value of $\varphi_{t}(p)$ in our implementation: we compute the value $z=\max _{t, v \in \mathcal{M}, i \in\{0,1\}}\left|T_{t, v}^{i}\right|$ measuring the maximum integer translation; then if $u^{*}(p)>0$, we replace $u^{*}(p)$ by $\left(u^{*}(p)+z\right)-z$; otherwise, we replace it by $\left(u^{*}(p)-z\right)+z$ instead. $v^{*}(p)$ is treated similarly. Then $\varphi_{t}(p)$ is set to $R^{\left(k_{p t}+k_{t}\right) \pi / 2} \varphi^{*}(p)+\left(T_{t, p}^{0}, T_{t, p}^{1}\right)^{T}$. Extraction of integer points and isolines is more robust with this rounding of $\varphi^{*}(p)$.

\subsection{Quadrangulation derived from parameterization}

While we enforce proper (zero) transitions across edges in the spanning tree, the resulting parameterization is globally valid if the following criteria are also satisfied:

- Seamlessness: the transition between two triangles across an edge that is not part of the spanning tree should have integer $\pi / 2$ rotation and translation;

- Injectivity: the local parameterization of each triangle $t \in \mathcal{T}$ should not be folded over, i.e., the parameterization $\varphi_{t}$ of every triangle $t$ should satisfy $\operatorname{det}\left(\nabla \varphi_{t}\right)>0$;

- Boundary condition: boundary and feature edges should align well with integer isolines.

Regular region. We thus define the regular region $\mathcal{M}_{R}$ of $\mathcal{S}$ as the set of faces of the original triangle mesh that are not adjacent to edges that either fail the seamlessness or the boundary conditions. Note that $\mathcal{M}_{R}$ typically covers most of the surface except a number of small patches (see Fig. 18 for various examples).

Quad extraction from parameterization. Within $\mathcal{M}_{R}$, we then extract all the points $c$ with half-integer parameter values, i.e., such that $\varphi(c)=\left(k_{1}+1 / 2, k_{2}+1 / 2\right)^{T}, k_{1}, k_{2} \in \mathbb{Z}$, as they correspond to quadrangle centers. After extraction of points with integer parameter values (which will form the vertices of our quad mesh), we establish the connectivity of each quadrangle by finding the four adjacent vertices from each half-integer center $c$. We discard any extracted quadrangle which has edges that cannot be traced through isolines (from one integer point to another), or has internal seams. For added robustness, we discard any quadrangle for which the isoline edge crosses an other integer isoline or feature or boundary. We are left with a quad mesh $Q_{R}$, with potentially small missing patches corresponding to places where our spanning parameterization failed. We use exact predicates [Shewchuk 1997] to determine if an integer point is in a triangle to avoid floating point errors.

Singular region. We now define the part of the surface $\mathcal{S}$ that is not covered by $Q_{R}$ as the singular region $\mathcal{M}_{S}=\mathcal{M} \backslash Q_{R}$. Note that $\mathcal{M}_{S} \cap \mathcal{M}_{R} \neq \emptyset$ since the regular region was not entirely covered with quadrangles (we discarded all quadrangles not fully contained in the regular region). We refine the original triangle mesh along the boundary $\partial Q_{R}$ to make sure we have a proper triangulation of the singular region $\mathcal{M}_{S}$. Even if the singular region did not inherit a proper parameterization from $\Psi$ (most of the time because of the presence of a singularity of the input frame field), we can now exploit the Morse function $\Psi^{0}$ in this area to extract a local Morsebased quadrangulation which, by construction, will exactly fill in the singular patches as illustrated in Fig. 7 and explained next. 


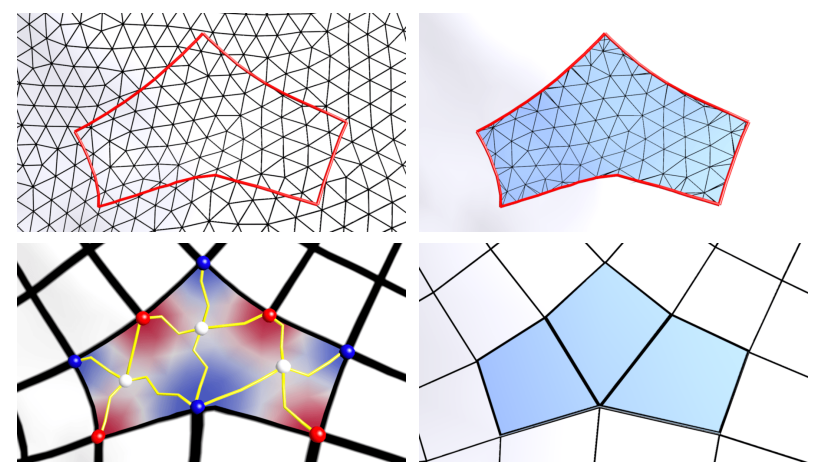

Fig. 7. Singular region. Top left: the boundary isolines in one local singular region. Top right: the tessellation of the singular region formed by the original mesh and the intersection points of the isolines with the original edges. Bottom left: the singular region with a quasi Morse-Smale complex, blue points are minima, red points are maxima, and white points are saddles. Bottom right: the resulting quadrangles.

\subsection{Quadrangulation of the singular region}

One of the main computational drawbacks of Morse-based quadrangulation is its need for a fine tessellation to capture all critical points. However, now that we have identified the few regions with singularities, we can locally refine the mesh in $\mathcal{M}_{S}$, and upsample $\Psi$ in order to guarantee a fast and proper Morse-based quadrangulation $Q_{S}$ of $\mathcal{M}_{S}$ that will enforce a seamless global quad mesh.

Local mesh refinement. The triangles within $\mathcal{M}_{S}$ are first refined through recursive mid-edge bisection: any edge with a length measured in the frame field induced metric $\left(F F^{T}\right)^{-1}$ that is greater than a threshold $\varepsilon_{e}$ is subdivided, thus splitting any adjacent triangle into two triangles. The subdivision ends when no edge is longer than $\varepsilon_{e}$ in $\mathcal{M}_{S}$ (we use $\varepsilon_{e}=0.5$ ). All subdivided faces inherit the frames of their original faces, and values of $\Psi$ are interpolated at the new vertices to provide a good first estimate of the periodic vector field. Once this refinement is performed, we further split in two any inner edge of $\mathcal{M}_{S}$ adjacent to boundaries or/and features, and any boundary/feature edge adjacent to corners: this last refinement step provides more degrees of freedom to extract the MSC properly.

Upsampling the periodic vector field. To ensure continuity with the regular region, the newly inserted vertices along the boundary $\mathcal{M}_{S} \cap Q_{R}$ are assigned boundary values $\Psi_{p}^{R}=\psi\left(\varphi_{p}\right)$ based on linear interpolation of the global parameterization in $\mathcal{M}_{R}$ to ensure exact continuity between the two regions. We then upsample the periodic vector field $\Psi$ within $\mathcal{M}_{S}$ by re-optimizing its internal values based on the input field and all the constraints, i.e., we solve the optimization of Eq. (18) over $\mathcal{M}_{S}$ with Gauss-Newton iterations, where we only add the linear constraints, $\Psi_{p}^{0}=\left(\Psi_{p}^{0}\right)^{R}, p \in \mathcal{M}_{S} \cap Q_{R}$, to match the new boundary values. The initial value of the refined $\mathcal{M}_{S}$ is obtained via $\psi \circ \varphi_{t}(p)$ for each vertex to provide a good guess based on the coarse solution. To guarantee robustness against badly shaped triangles (which can appear when we cut out the singular region from the original mesh), we further modify the term $|t| / 2\left|\mathbf{e}_{p q}\right|^{2}$ in Eq. (15) for every edge $\mathbf{e}_{p q}$ with length smaller than $\varepsilon_{s}$ into $|t| / 2\left(\left|\mathbf{e}_{p q}\right|+\varepsilon_{s}\right)^{2}$ for $\varepsilon_{s}=\bar{e} / 100$ in our implementation $(\bar{e}$ being the average edge length in $\mathcal{M}_{S}$ ). After the optimization has converged, the result is a finely-discretized $4 \mathrm{D}$ vector field $\Psi$ within the singular region which matches, by construction, the original field along the boundary.

Existence of a fill-in quadrangulation. The quadrangulation that we wish to extract from the singular region through a Morse-Smale complex must be compatible with (i.e., precisely fit) the quadrangulation $Q_{R}$ already extracted from the regular region. The quadrangulation $Q_{S}$ of $\mathcal{M}_{S}$ can be obtained from the quasi-dual MSC of $\left.\Psi^{0}\right|_{\mathcal{M}_{S}}$ if the two following conditions hold:

- Any two adjacent vertices of $\mathcal{M}_{S}$ have distinct values: this is a necessary condition for Morse functions;

- Any boundary max/min vertex (i.e., with a $\Psi^{0}$ value higher/lower than its two neighbors along the boundary $\partial \mathcal{M}_{S}$ ) is also a max/min of its one-ring in $\mathcal{M}_{S}$ : this will enforce that $\partial \mathcal{M}_{S}$ will exactly correspond to quad edges after Morse extraction.

The first condition is automatically satisfied along $\mathcal{M}_{S} \cap Q_{R}$ since the values of the Morse function $c c(u, v)$ on $\partial Q_{R}$ (which, by construction, had a non-degenerate parameterization) were used as boundary conditions; for the remainder of the domain $\mathcal{M}_{S} \backslash Q_{R}$, we perform local perturbation of each pair of adjacent vertices with the same value after optimization to remove all degeneracies.

The second condition can then be enforced as follows. First, the newly-optimized Morse function $\Psi^{0}$ in $\mathcal{M}_{S} \backslash Q_{R}$ is replaced by $c c\left(\varphi^{*}(p)\right)$ (see Eq. (19)). Note that these two expressions should be strictly equal if exact arithmetics was used and if the optimized $\Psi$ exactly satisfied the imposed constraints; but given the presence of floating-point operations and the use of an inexact optimization, this "reprojection" guarantees we bring the Morse function exactly in the interval $[-1,1]$. Second, every local minimum (resp., maximum) along $\partial \mathcal{M}_{S}$ is set to $-(1+\varepsilon)$ (resp., $1+\varepsilon$ ), see Fig. 19. This change does not alter the continuity across the regular and singular regions (i.e., along $\mathcal{M}_{S} \cap Q_{R}$ ) since the boundary extrema remain extrema; but this perturbation of the extremal values now guarantees that the second condition mentioned above is satisfied. With this procedure (for which we provide a more detailed analysis in App. A), we efficiently bring robustness to the final stage: the extraction of the quad mesh of $\mathcal{M}_{S}$.

Quadrangle extraction. We use Morse-based extraction of quadrangles to find the quadrangulation $Q_{S}$ of the singular region. Note that our initial triangulation of $\mathcal{M}_{S}$ (by cutting the mesh along border isolines of $Q_{R}$, see Fig. 7) may have generated nearly degenerate triangles, especially when the input was very coarse compared to the frame-induced metric; prior to quad extraction, we thus perform a global subdivision with a threshold $\varepsilon_{g}=2$ under the metric $\left(F F^{T}\right)^{-1}$. After this last safety measure, the Morse extraction produces proper quadrangles since this region (made out of multiple connected components) have been locally refined based on edge length, hence enforcing the validity of the quasi-dual MSC of the $c c$ component of $\Psi$ resulting from the local re-optimization described above. Note that we treat each feature as boundary; local remeshing (see supplemental material), relaxation and/or projection of the quad vertices onto the original mesh can be done as well if needed. 

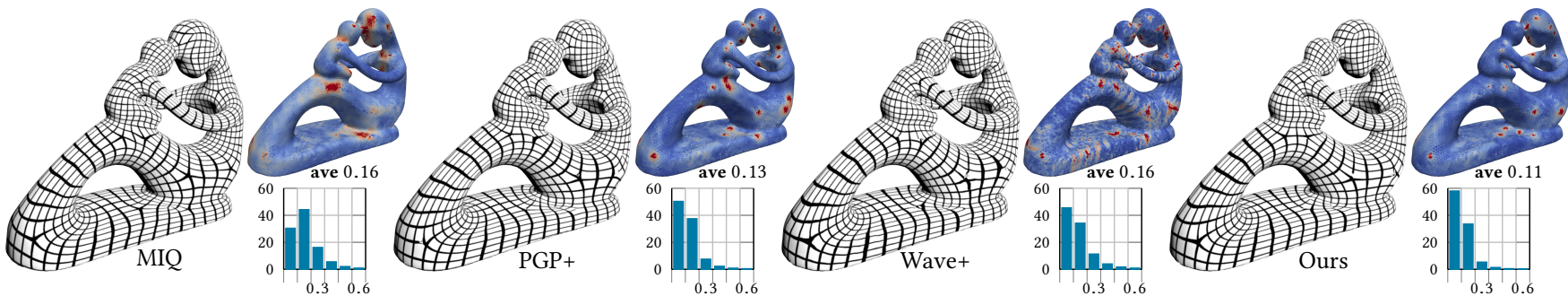

Fig. 8. Advantages of 4D vector field. The models with texture show the parameterization of MIQ and the reconstructed parameterizations of $\mathrm{PGP}+/$ Wave+/Ours. The color (blue 0 to red $0.6,>0.6$ shown with red) indicates frame alignment distortion of these parameterizations. The histograms are the area percentage distributions of frame alignment distortion.

\section{RESULTS}

We now discuss how our approach compares to existing quadrangulation methods in terms of both quality and timings of the results. We used input frame fields computed through the metric customized method of Jiang et al. [2015]. Unless otherwise specified, our frame fields were curl-corrected using [Zhang et al. 2010]. To quantify the quality of our results, we use the commonly used scaled Jacobian (S.J., see [Knupp 2000]) computed on the resulting quad meshes, and provide average metric distortion $\left(\mathcal{D}_{m}\right)$ and average frame alignment distortion $\left(\mathcal{D}_{f}\right)$ computed as

$$
\begin{aligned}
& \mathcal{D}_{t, f}=\min _{k \in \mathbb{Z}}\left\|\nabla \varphi_{t} \cdot F_{t}-R^{k \pi / 2}\right\|, \mathcal{D}_{f}=\frac{1}{|\mathcal{M}|} \sum_{t \in \mathcal{M}} \mathcal{D}_{t, f}|t| ; \\
& \mathcal{D}_{t, m}=\min _{R \in S O(2)}\left\|\nabla \varphi_{t} \cdot F_{t}-R\right\|, \quad \mathcal{D}_{m}=\frac{1}{|\mathcal{M}|} \sum_{t \in \mathcal{M}} \mathcal{D}_{t, m}|t| .
\end{aligned}
$$

Besides the results we present here, a total of over 140 models (including those from [Myles et al. 2014]) are shown in the supplemental material, along with their relevant statistics.

\subsection{Comparisons}

Specific comments are called for when comparing our approach to previous works. We provide a detailed discussion of the differences between main prior methods and our work next.

PGP. We also compared our approach to PGP [Ray et al. 2006]. In order to get a parameterization similar to ours, we chose $k \pi$ as isolines in the PGP formulation; that is, we picked:

$$
(\cos (\pi u), \sin (\pi u), \cos (\pi v), \sin (\pi v))
$$

as variables, and recovered the global parameterization with our method (denoted as 'PGP+' hereafter). Fig. 8 demonstrates that our approach enforces frame field alignment much better than PGP formulation. Our hybrid approach also benefits from an additional advantage: our formulation has a larger solution space due to the use of a $4 \mathrm{D}$ vector field, helping with alignment to feature and boundary constraints. Indeed, with $k \pi$ as isolines, PGP must enforce $\cos \left(\pi\left(u+T_{1}\right)\right)=\cos (\pi u)$ and $\cos \left(\pi\left(v+T_{2}\right)\right)=\cos (\pi v)$ (i.e., $T_{1}, T_{2} \in 2 \mathbb{Z}$ ) to take care of transitions, while we only need $T_{1}$ and $T_{2}$ to have the same parity-which is a sufficient and necessary condition for Morse-based quad meshing of a simply connected domain. Moreover, we use $\sin (\pi u) \sin (\pi v)=0$ to formulate feature and boundary alignments, while PGP cannot directly formulate these constraints. Moreover, our method is guaranteed to produce pure quad meshes while PGP offers no guarantee of a parameterizations leading to pure quad meshes.

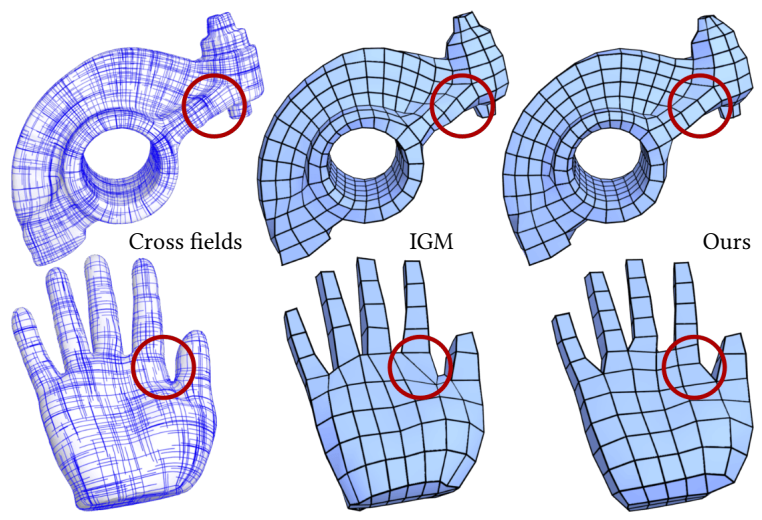

Fig. 9. Comparison with IGM. For input meshes and cross fields from IGM [Bommes et al. 2013a] (top: rockerarm model; bottom: hand model), our results exhibit better alignment.

MIQ \& IGM. The MIQ approach [Bommes et al. 2009] and its followup, IGM [Bommes et al. 2013a], offer high quality quad meshes as well. Compared with these two methods, our hybrid approach shows a better alignment to input frame fields, but at the price of slightly more singularities to reduce the frame alignment/metric distortion, see Figs. 8 and 9. Importantly, our method can generate valid quad meshes even when the input frame field and features do not satisfy pure-quad holonomy conditions, while these two methods fail to get valid quad meshes as they keep the input singularity structure, see Figs. 4 and 10: as shown in the first column of Fig. 10, MIQ results in fold-overs and degenerated faces, while [Ebke et al. 2013] cannot extract valid quad meshes. MIQ can obtain valid results when no feature alignment is requested (second column in Fig. 10). We also compare our method with IGM [Bommes et al. 2013a] by using cross fields from their paper, see Fig. 9: our method applied to curl-corrected cross fields (using [Zhang et al. 2010]) results in meshes with better orientation alignment than IGM.

Wave-based approach. Our method was designed to handle even very coarse meshes as input. A result of our hybrid approach using the coarse

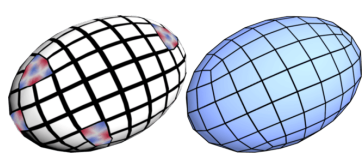
ellipse mesh shown in Fig. 2 as input is shown in the inset (left: parameterization vs. Morse regions; right: final quad mesh). The wave-based methods [Zhang et al. 2010], instead, need much denser meshes as input to extract a reasonable quasi-dual Morse-Smale complexes, see Figs. 2 and 17. Our formulation based on covariant 


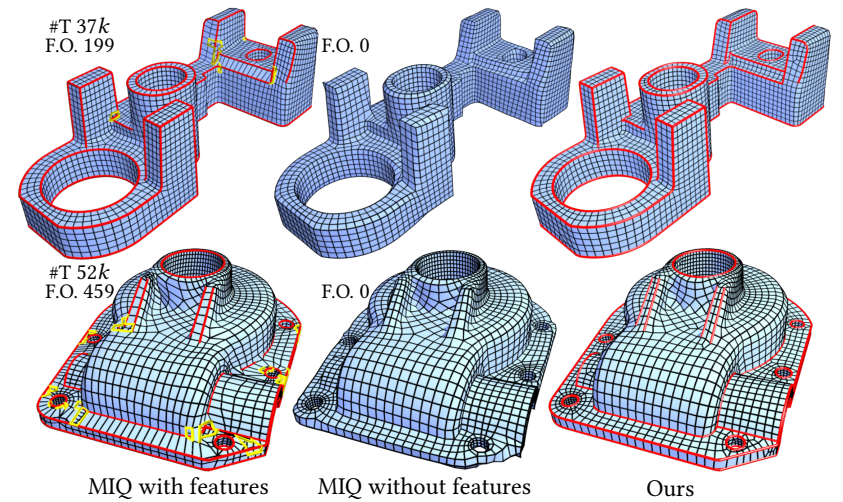

Fig. 10. Comparison with MIQ. The Cad5 and Cast models have intricate features. Our results are on the right, with red curves indicating feature lines. MIQ results are on the left and middle columns, with cells extracted by libQEx [Ebke et al. 2013]; the left column with feature constraints shows holes (marked with yellow curves) due to fold-overs and degenerate faces (F.O. is the number of fold-over triangles, \#T is the triangle count of the input); the middle column does not respect feature constraints.

derivative can get a parameterization that aligns to the input frame field significantly better as well. To quantify this statement, we reconstructed a parameterization based on the energy formulation in [Zhang et al. 2010] (denoted as 'Wave+' in Fig. 8) to compare with ours, and alignment distortion was indeed notably worse. We also compared on nine models, each having three different triangle density (from coarse (a) to dense (c), see Fig. 11). On coarse and medium meshes, the wave-based approach completely fails to get a frame-aligned quad mesh as expected; instead, our method always returns quad meshes that are visually comparable (see Fig. 17 for an illustration on the Pig model) to the wave-based results using the dense inputs, with already speedups of up to a factor 10 on these models with at most $40 \mathrm{~K}$ triangles. For dense meshes, our running times are similar to the wave-based approach; note that our timings include the reoptimization of the periodic vector field over singular regions, which is actually unnecessary for dense enough inputs.

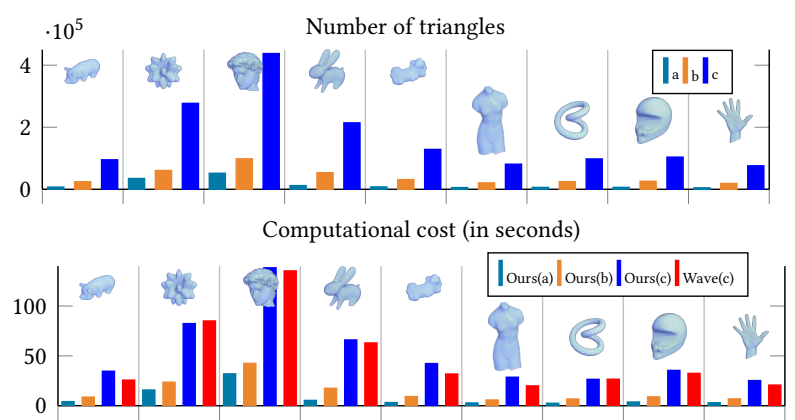

Fig. 11. Comparison with wave-based method. Statistics for 9 input models at 3 levels of refinement. The wave approach only finds good quad meshes for the finest inputs (see supplemental material for additional info).

Instant field-aligned meshing. When compared to [Jakob et al. 2015], Fig. 12 demonstrates that our approach better preserves features, resulting in a much lower Hausdorff error distance $(0.43 \%$ vs. $1.04 \%$ with respect to the bounding box diagonal). While their
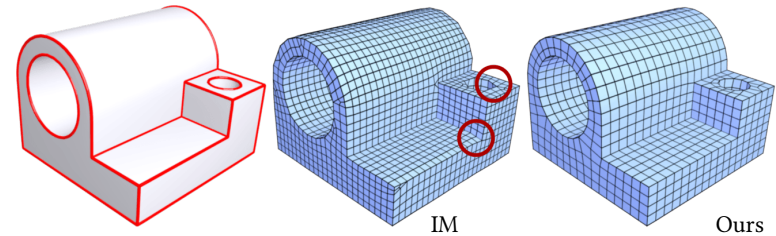

Fig. 12. Comparison with Instant Meshing. Left to right: input mesh with sharp features (red), result from [Jakob et al. 2015], and our result. Our approach preserves features better, and can construct coarser quad meshes.

work requires less computation than ours, it is limited to input cross-frame fields with isotropic metrics, and only guarantees quaddominant meshes (which can of course be subdivided to create pure quad meshes, at the price of losing alignment to the input field).

Controlled-distortion parameterization. The approach of Myles and Zorin [2013] can be used to generate quad meshes, but does not incorporate the typical constraints needed for meshing such as direction and sizing fields; instead, it looks for a conformal metric with cone singularities so that the resulting parameterization best aligns with a set of features. Despite the significant differences in meshing goals with our approach, we provide in Fig. 14 some examples to discuss the pros and cons of both approaches. From a set of features, we used [Jiang et al. 2015] to design a uniform cross field (with 16 singularities). We then curl-correct the input frame field using [Zhang et al. 2010], and compare the results of our approach to [Myles and Zorin 2013] (we target a fine quadrangulation density as their approach would fail to provide low distortion otherwise). If only a small correction of the curl is done, our approach returns a mesh with low metric distortion (measured with respect to the original cross field), but a hundred singularities due to the presence of curl in the field. Inversely, a more aggressive curl correction makes for a factor two reduction in singularities, but at the price of a higher metric distortion obviously. In contrast, the unguided approach of [Myles and Zorin 2013] finds a mesh with low metric distortion and low singularity count. This is to be expected: because our approach takes an input frame field and consider both direction and metric alignment simultaneously, it does not target distortionminimizing parameterization like [Myles and Zorin 2013] seeks, but low frame-alignment distortion. For quad meshing, we argue that this latter goal is most relevant in practice: the ability to provide guidance through an arbitrary input field is a necessity.

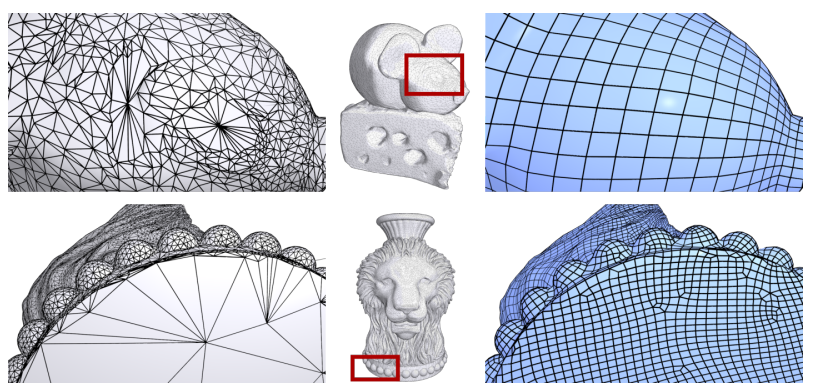

Fig. 13. Robustness to input mesh quality. Even on models with bad triangles (top: mouse cheese; bottom: lion vase), our approach produces non-degenerate quadrangulations (left: local input; right: output quads). 


\subsection{Solver performance}

In Fig. 15, we quantify the benefits of having a simple way to initialize our 4D vector field optimization Eq. (16) via an eigensolver: we compare the evolution of the energy across Gauss-Newton steps for an arbitrary initial value vs. our eigensolver-based initialization. For every model we tried, the prior eigensolver saves between a factor two and twenty in terms of number of steps before convergence (where a threshold of $1 e-3$ on gradient magnitude is used), and the final energy value is up to 5 times lower, thus reaching much better field alignment as shown in Fig. 16.

\subsection{Robustness to irregular sampling}

We also tested the robustness of our quad meshing with respect to input mesh density and regularity. Fig. 17 demonstrates that we get similar results for meshes of the same shape with different density obtained via mid-edge bisections of an initial mesh: our connectionbased smoothness energy is by and large insensitive to the input mesh quality. Fig. 13 confirms that even on input triangular meshes with high aspect ratio and wildly varying sizes, our method still generates high-quality quad meshes. Note, however, that long and large triangles in the input can lead to larger singular region, as indicated in Tab. 1 for the vase lion and the hand: the spanning parameterization is less likely to handle these cases well.

\subsection{Frame field control}

Finally, we evaluate the ability of our approach to control alignment to an arbitrary input frame field and complex features.

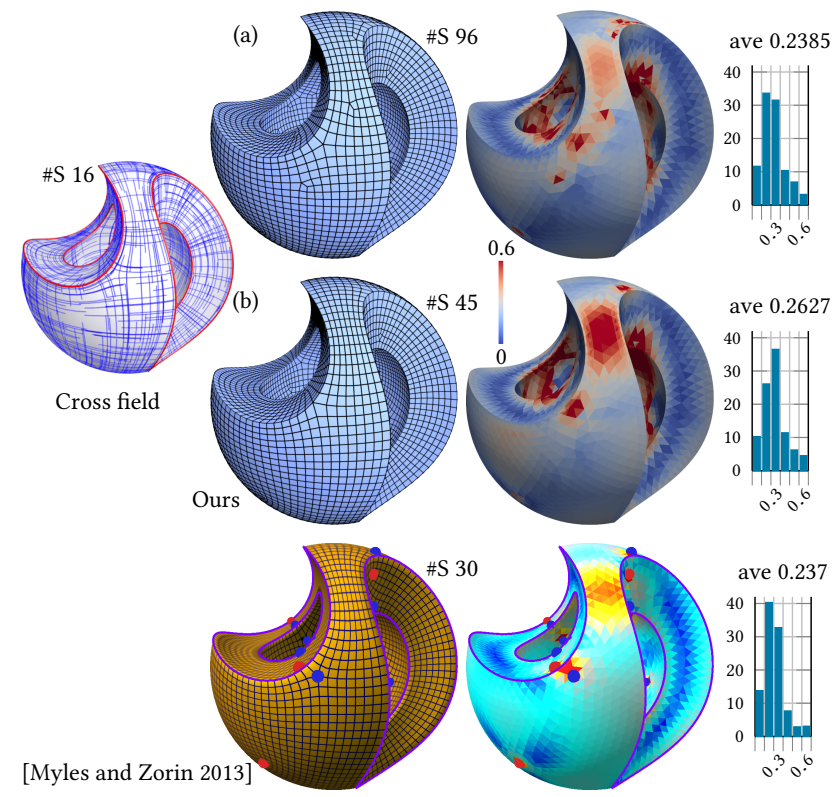

Fig. 14. Sculpt mesh. From an input frame field, we generate two frame fields using little (row (a)) or significant (row (b)) curl correction [Zhang et al. 2010]. We show the respective output meshes (left) and their associated metric distortions with respect to the original cross field (right), as well as the result of [Myles and Zorin 2013] (bottom), along with its distortion histogram. \#S denotes the number of singularities present in the quad mesh.
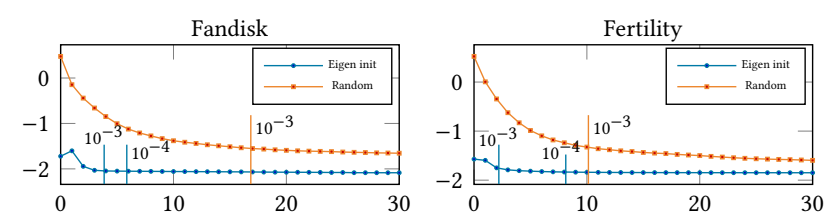

Fig. 15. Solver convergence. Evolution of energy $\mathcal{E}$ (y-axis in log scale) against Gauss-Newton steps, for an arbitrary vs. eigenbased initialization. The vertical lines marked $10^{-3}$ or $10^{-4}$ indicate the iteration for which the gradient norms fall below these thresholds.

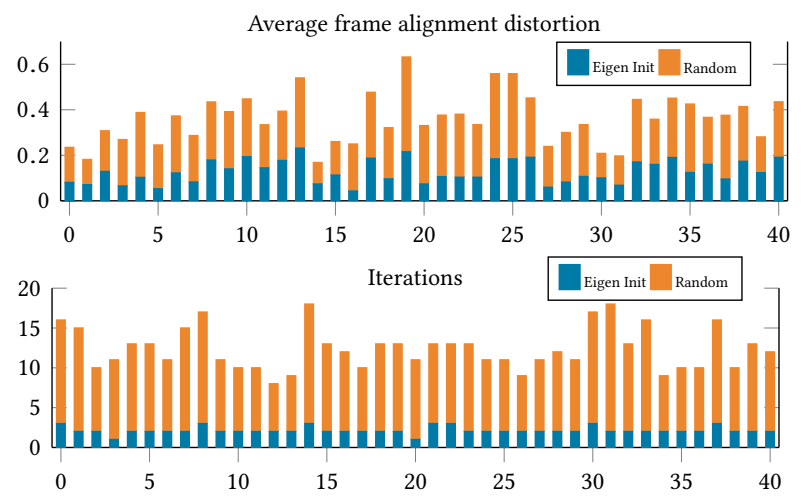

Fig. 16. Solver quality and efficiency. Comparisons of alignment quality and number of solver iterations (until convergence, tested via gradient magnitude) for random vs. eigenbased initializations over 41 meshes.

Frame and feature alignment. As shown throughout the figures of this paper and in the supplemental material, we tried over 140 models of various complexity with cross or arbitrary frame fields, and our solver always managed to accommodate the implied direction and length constraints, resulting in quad meshes that follow the frame fields closely, see Fig. 3 for instance. Inconsistencies between directions and sizing are also handled automatically. We refer the reader to Tab. 1 for quantitative evaluations, where we also evaluate the Hausdorff distance between input and output feature lines to measure feature preservation.

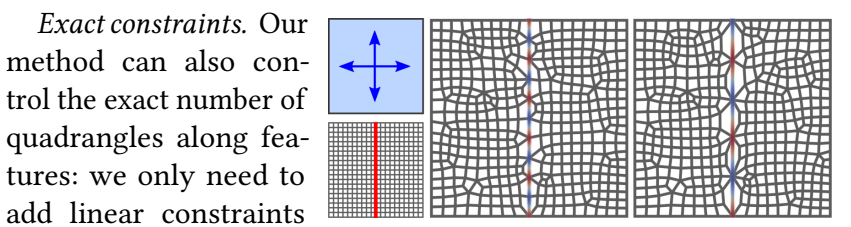
on $\Psi_{p}^{0}$ for each vertex along features. This is demonstrated in the inset, where the red line is the feature line; the initial result (bottom left) without constraints for a constant frame field can be modified to have 11 (middle) or 6 (right) vertices regularly spaced along the feature. Using frame fields compatible with these constraints would dramatically improve the quality of the results, obviously.

Robustness to complex features. We also demonstrate the robustness of our approach by testing it on complex mechanical parts and extreme models in Figs. 1 and 18. None of the hundreds of meshes we tried made our method fail. 
Table 1. Statistics. We list relevant statistics for the models used in this paper. $\# T$ is the number of input triangles, $\# Q$ is the number of output quads. $\# S(Q)$ indicates the number of singularities in the resulting quad mesh, while $\# S(F)$ is for the input fields. S.J. is the average scaled Jacobian of quadrangles. MSC.R. is the percentage of singular area covered by MSC. $\mathcal{D}_{m}$ is the average distortion of metric, $\mathcal{D}_{f}$ is that of frame alignment. The $\mathcal{D}_{m}$ and $\mathcal{D}_{f}$ about sculpt (a) and (b) are based on the original cross field. $D_{f l}$ is the Hausdorff distance between input and output feature lines, given as a percentage of the bounding box diagonal.

\begin{tabular}{|c|c|c|c|c|c|c|c|c|c|c|}
\hline Models & $\# \mathrm{~T}$ & $\# Q$ & $\# \mathrm{~S}(\mathrm{~F})$ & $\# \mathrm{~S}(\mathrm{Q})$ & S.J. & Time & MSC.R. & $\mathcal{D}_{m}$ & $\mathcal{D}_{f}$ & $D_{f l}$ \\
\hline \begin{tabular}{|l|} 
Aircraft(Fig. 3) \\
\end{tabular} & 24920 & 3807 & 54 & 87 & 0.945 & 9.07 & 5.97 & \begin{tabular}{|l|}
0.075 \\
\end{tabular} & 0.086 & 0.27 \\
\hline Beetle(Fig. 6) & 42468 & 2559 & 60 & 76 & 0.983 & 12.79 & 8.78 & 0.086 & 0.097 & 0.44 \\
\hline Part(Fig. 3) & 26118 & 2470 & 20 & 44 & 0.976 & 7.62 & 3.9 & 0.094 & 0.104 & 0.55 \\
\hline Fandisk & 30078 & 816 & 32 & 66 & 0.973 & 8.57 & 6.88 & 0.127 & 0.143 & 0.45 \\
\hline Cheese & 389972 & 34291 & 2270 & 2472 & 0.965 & 156.4 & 15.89 & 0.152 & 0.171 & 0.32 \\
\hline Nautilus & 50438 & 4952 & 74 & 155 & 0.983 & 17.02 & 4.55 & 0.118 & 0.126 & 0.25 \\
\hline Carter & 79310 & 8186 & 252 & 308 & 0.98 & 31.7 & 6.82 & 0.098 & 0.108 & 0.35 \\
\hline Cover & 140914 & 21278 & 189 & 669 & 0.981 & 49.1 & 5.03 & 0.098 & 0.11 & 0.19 \\
\hline Datatech & 130956 & 15107 & 212 & 520 & 0.979 & 52.36 & 7.62 & 0.118 & 0.131 & 0.29 \\
\hline Deckel & 2962 & 2950 & 62 & 86 & 0.986 & 14.2 & 6.32 & 0.086 & 0.095 & 0.76 \\
\hline Fuse & 107050 & 9050 & 258 & 451 & 0.965 & 40.4 & 7.69 & 0.127 & 0.14 & 0.2 \\
\hline Mouse & 8708 & 6167 & 209 & 323 & 0.958 & 14.7 & 13.8 & 0.123 & 0.142 & - \\
\hline Vase-lion(Fig. 13) & 100000 & 23358 & 1296 & 2534 & 0.911 & 97.0 & 28.7 & 0.192 & 0.221 & - \\
\hline Cad5 & 37336 & 4919 & 76 & 134 & 0.987 & 12.3 & 5.63 & 0.09 & 0.099 & 0.27 \\
\hline Cad5 $(\mathrm{m}$ & 37336 & 4893 & 76 & 76 & 0.988 & 28 & - & 0.09 & 0.10 & \\
\hline Cast & 52388 & 5089 & 104 & 201 & 0.978 & 19.3 & 7.91 & 0.097 & 0.107 & 0.36 \\
\hline Cast(mid Fig. 10) & 52388 & 5138 & 104 & 102 & 0.98 & 96 & - & 0.114 & 0.12 & \\
\hline Fertil & 954 & 2248 & 54 & 92 & 0.959 & 10 & 9.43 & 0.1 & 0.11 & - \\
\hline Ferti & 7954 & 2283 & 54 & 54 & 0.946 & 30 & - & 0.15 & 0.16 & - \\
\hline Fertili & 27954 & 2289 & 54 & 90 & 0.957 & 15 & 8.66 & 0.11 & 0.13 & - \\
\hline Fertility(Wave + ) & 27954 & 2271 & 54 & 89 & 0.959 & 9 & 9.91 & 0.14 & 0.16 & - \\
\hline hand & 8480 & 176 & 40 & 34 & 0.969 & 3.5 & 24.76 & \begin{tabular}{|l|}
0.133 \\
\end{tabular} & 0.146 & - \\
\hline hand(IGM) & 8480 & 180 & 40 & 40 & 0.924 & - & - & - & - & - \\
\hline rockerarm & 70318 & 499 & 30 & 38 & 0.973 & 32 & 12.17 & 0.084 & 0.107 & - \\
\hline rockerarm(IGN & 70318 & 500 & 30 & 30 & 0.958 & 11.9 & - & - & - & - \\
\hline Joint & 2096 & 1764 & 24 & 26 & 0.996 & 6.54 & 2.34 & \begin{tabular}{|l|}
0.049 \\
\end{tabular} & 0.052 & 0.38 \\
\hline Joint(IM) & 20968 & 3222 & 24 & 56 & 0.991 & $<1$ & & - & 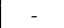 & - \\
\hline pig(a,ours) & 6973 & 3054 & 40 & 70 & 0.9767 & 4.12 & 6.687 & 0.0596 & 0.0662 & - \\
\hline , ours) & 24229 & 3064 & 40 & 68 & 0.9756 & 8.64 & 5.017 & 0.0578 & 0.066 & - \\
\hline pig(c,ours) & 94937 & 3067 & 40 & 74 & 0.9756 & 34.6 & 3.981 & 0.0616 & 0.0704 & - \\
\hline pig(a,wave) & 6973 & 622 & 40 & 371 & 0.7333 & 2.02 & 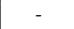 & 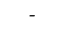 & 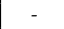 & - \\
\hline pig(b,wave) & 24229 & 2927 & 40 & 778 & 0.8969 & 5.15 & - & 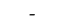 & - & - \\
\hline pig(c,wave) & 94937 & 3084 & 40 & 82 & 0.9752 & 25.7 & 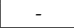 & 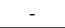 & - & - \\
\hline & 22070 & 5890 & 16 & 45 & 0.995 & 8.6 & 2.046 & $0.263^{*}$ & $0.265^{*}$ & \\
\hline sculpt(b) & 22360 & 5913 & 16 & 96 & 0.992 & 8.4 & 2.909 & $0.238^{*}$ & $0.242^{*}$ & - \\
\hline sculpt(Myles[2013]) & 7342 & 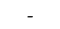 & - & 30 & 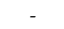 & 16.5 & - & 0.237 & - & \\
\hline
\end{tabular}

Supplemental Material. Lastly, we provide a series of results to offer additional evaluations of our technique. Our results for over 140 models and frame fields are listed, along with accompanying statistics. We also provide timings to show that our method is orders of magnitude faster than previous Morse-based methods.

\section{CONCLUSION}

We presented an efficient and robust quadrangulation method that can be controlled by a guiding frame field and allows feature alignment constraints. We designed our method to be both efficient and robust so as to generate quad meshes with complicated features: by mixing Morse-based and parameterization-based quad extraction based on a $4 \mathrm{D}$ vector field derived from the input frame field, our approach guarantees quad meshes with non-degenerate cells that closely match user constraints. The quadrangulation process is significantly faster than previous Morse-based methods as our nonlinear solver efficiently exploits an eigenbased approximation of the optimal 4D field and our extraction of quadrangles near singularities is driven by a Morse function which only requires very localized refinements of the input mesh. The resulting Morse-Parameterization hybrid approach has the unique property of being simultaneously
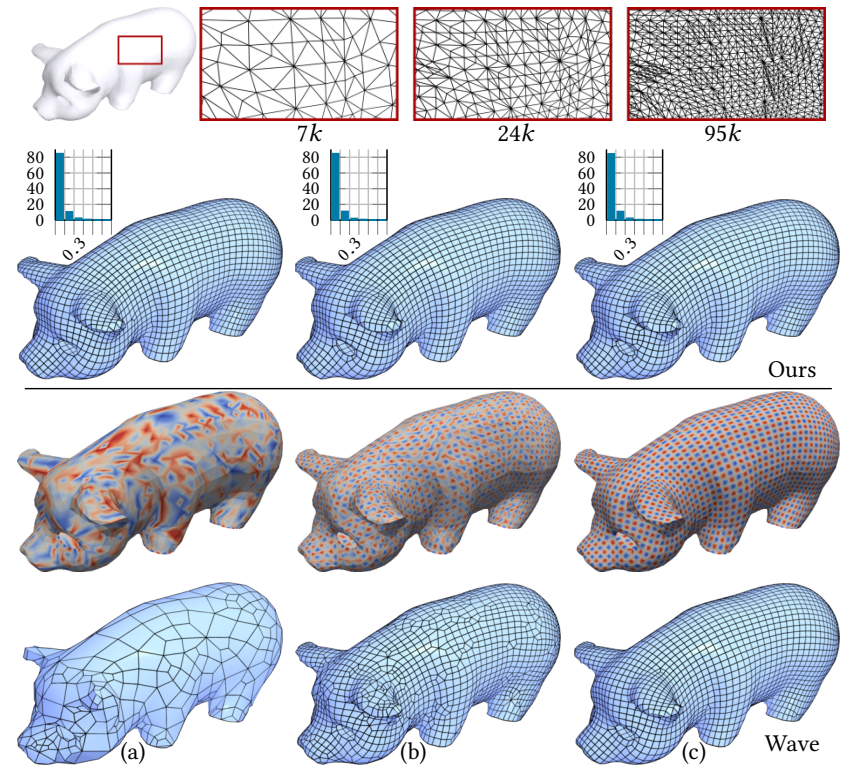

Fig. 17. Robustness to input triangle density. Top: our approach is mostly insensitive to the density of triangles in the input: for three different inputs of the same geometry (see close-ups), our approach produces similar quad meshes (histograms indicate area distribution of frame alignment distortion). Bottom: in contrast, the results of wave-based method [Zhang et al. 2010] are widely different on these three inputs, because the Morse function is not properly captured if the mesh is not dense enough locally.

fast and robust while guaranteeing valid, pure quad meshes as output. We generate high-quality meshes with low frame alignment distortion compared to classical methods such as MIQ or PGP. All quads are guaranteed to be non-degenerate, i.e., there always exits a one-to-one mapping from an element to the unit square.

Still, our approach may generate poor quality quadrangles on rare occasions, as shown in the inset: two adjacent edges of the (blue) quad are almost collinear and along a (red) feature. Just like all other

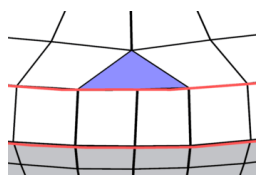
methods targeting frame field alignment, we cannot guarantee that all quads have positive scaled Jacobian. While we can split these suboptimal quads or perform local remeshing like many other meshing methods do in practice, finding a preemptive way to guarantee the absence of such quadrangles (perhaps by adding a "padding" layer of quadrangles around feature lines) would be a nice extension.

We also believe that our implementation can be further accelerated. Frame field based methods have been made very efficient by exploiting a multigrid approach [Ebke et al. 2016], which could benefit our approach. Moreover, the refinement of singular regions can be made in parallel for each disconnected component of the singular region (instead of serially in our implementation), which should reduce computational costs quite drastically. Treating singular regions using ideas given in [Takayama et al. 2014] may also offer a novel way to replace our current MSC-based approach.

Providing more control over the number and placement of singularities that had to be added in order to form a quad mesh would be 


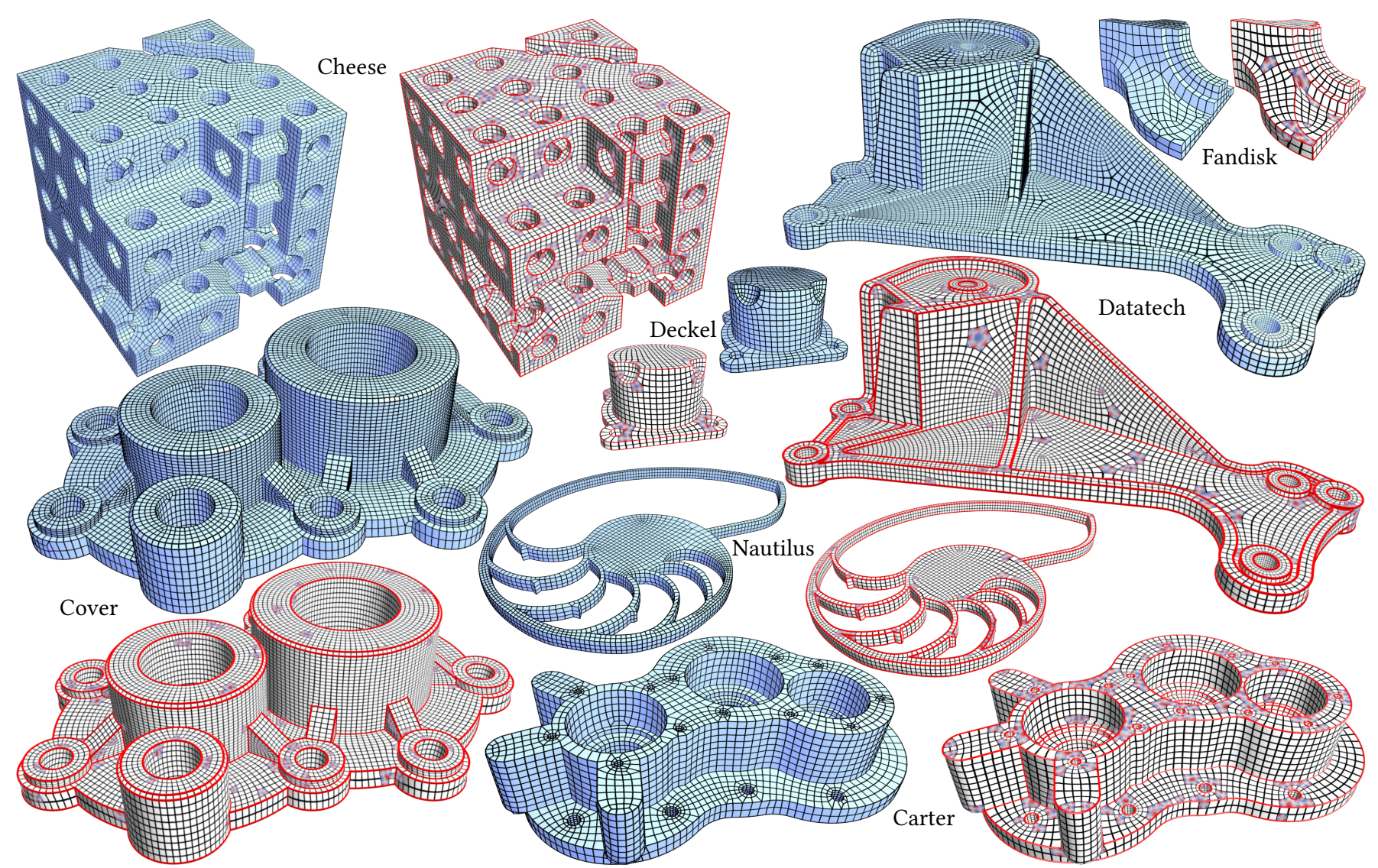

Fig. 18. Gallery. Collection of results of our hybrid approach to quadrangulation on a variety of surface models, including particularly difficult geometric domains due to the complexity of their shape, feature curves and topology. For each example, we show the Morse function in refined patches within the spanning parameterization that was computed, and the final quad mesh in blue.

a valuable extension. Knowledge of symmetry between different regions of the input geometry can also be exploited in our framework. Another very valuable future work is the extension of our method to 3D for practical hex remeshing. Because 3D Morse-Smale Complex can contain non-hex cell, we cannot expect pure hexahedral results as shown in [Ling et al. 2014]-even more so given that additional topological conditions on singular regions are required for the existence of fill-in hex meshes [Eppstein 1999; Mitchell 1996]. However, if hex-dominant meshes are sought after, such a 3D extension may be viable due to its robustness.

\section{ACKNOWLEDGMENTS}

We would like to thank the anonymous reviewers for their comments and suggestions. This work was partially supported by NSFC (No. 61522209, 61210007) and Fundamental Research Funds for the Central Universities (2018FZA5011). MD gratefully acknowledges the INRIA International Chair program, and Zhejiang University for hosting him superbly well during the final editing of this work. YT acknowledges partial support from NSF (CCF-1655422 and DMS1721024). The 3D models in this paper and its supplemental material were provided by the AIM@SHAPE-VISIONAIR Repository, the INRIA Gamma team and the Stanford 3D Scanning Repository.

\section{REFERENCES}

David Bommes, Marcel Campen, Hans-Christian Ebke, Pierre Alliez, and Leif Kobbelt. 2013a. Integer-grid Maps for Reliable Quad Meshing. ACM Trans. Graph. 32, 4, Article 98 (2013).

David Bommes, Bruno Lévy, Nico Pietroni, Enrico Puppo, Claudio Silva, Marco Tarini, and Denis Zorin. 2013b. Quad-Mesh Generation and Processing: A Survey. Comput. Graph. Forum 32, 6 (2013), 51-76.

David Bommes, Henrik Zimmer, and Leif Kobbelt. 2009. Mixed-Integer Quadrangulation. ACM Trans. Graph. 28, 3, Article 77 (2009).

Marcel Campen, David Bommes, and Leif Kobbelt. 2015. Quantized Global Parametrization. ACM Trans. Graph. 34, 6, Article 192 (2015).

Keenan Crane, Mathieu Desbrun, and Peter Schröder. 2010. Trivial Connections on Discrete Surfaces. Comput. Graph. Forum 29, 5 (2010), 1525-1533.

Fernando de Goes, Mathieu Desbrun, and Yiying Tong. 2015. Vector Field Processing on Triangle Meshes. In ACM SIGGRAPH Asia 2015 Courses. Article 17.

Olga Diamanti, Amir Vaxman, Daniele Panozzo, and Olga Sorkine-Hornung. 2015. Integrable PolyVector Fields. ACM Trans. Graph. 34, 4, Article 38 (2015).

Shen Dong, Peer-Timo Bremer, Michael Garland, Valerio Pascucci, and John C. Hart. 2006. Spectral Surface Quadrangulation. ACM Trans. Graph. 25, 3 (2006), 1057-1066.

Hans-Christian Ebke, David Bommes, Marcel Campen, and Leif Kobbelt. 2013. QEx: Robust Quad Mesh Extraction. ACM Trans. Graph. 32, 6, Article 168 (2013).

Hans-Christian Ebke, Patrick Schmidt, Marcel Campen, and Leif Kobbelt. 2016. Interactively Controlled Quad Remeshing of High Resolution 3D Models. ACM Trans. Graph. 35, 6, Article 218 (2016).

Herbert Edelsbrunner, John Harer, and Afra Zomorodian. 2003. Hierarchical MorseSmale Complexes for Piecewise Linear 2-Manifolds. Discrete Comput. Geom. 30, 1 (2003), 87-107.

David Eppstein. 1999. Linear complexity hexahedral mesh generation. Computational Geometry 12, 1-2 (1999), 3-16. 
Jin Huang, Muyang Zhang, Jin Ma, Xinguo Liu, Leif Kobbelt, and Hujun Bao. 2008 Spectral Quadrangulation with Orientation and Alignment Control. ACM Trans. Graph. 27, 5, Article 147 (2008).

Wenzel Jakob, Marco Tarini, Daniele Panozzo, and Olga Sorkine-Hornung. 2015. Instant Field-aligned Meshes. ACM Trans. Graph. 34, 6, Article 189 (2015).

Tengfei Jiang, Xianzhong Fang, Jin Huang, Hujun Bao, Yiying Tong, and Mathieu Desbrun. 2015. Frame Field Generation Through Metric Customization. ACM Trans. Graph. 34, 4, Article 40 (2015).

Felix Kälberer, Matthias Nieser, and Konrad Polthier. 2007. QuadCover: Surface Parameterization using Branched Coverings. Comp. Graph. Forum 26, 3 (2007), 375-384.

Felix Kälberer, Matthias Nieser, and Konrad Polthier. 2011. Stripe Parameterization of Tubular Surfaces. In Topological Methods in Data Analysis and Visualization: Theory Algorithms, and Applications, Valerio Pascucci, Xavier Tricoche, Hans Hagen, and Julien Tierny (Eds.). Springer Berlin Heidelberg, 13-26.

Felix Knöppel, Keenan Crane, Ulrich Pinkall, and Peter Schröder. 2015. Stripe Patterns on Surfaces. ACM Trans. Graph. 34, 4, Article 39 (2015)

Patrick M. Knupp. 2000. Achieving finite element mesh quality via optimization of the Jacobian matrix norm and associated quantities. Part II-A framework for volume mesh optimization and the condition number of the Jacobian matrix. Internat. 7 Numer. Methods Engrg. 48, 8 (2000), 1165-1185.

Yu-Kun Lai, Miao Jin, Xuexiang Xie, Ying He, Jonathan Palacios, Eugene Zhang, ShiMin $\mathrm{Hu}$, and Xianfeng Gu. 2010. Metric-Driven RoSy Field Design and Remeshing. IEEE Trans. Vis. Comput. Graph. 16, 1 (2010), 95-108.

Ruotian Ling, Jin Huang, Bert Jüttler, Feng Sun, Hujun Bao, and Wenping Wang. 2014. Spectral Quadrangulation with Feature Curve Alignment and Element Size Control. ACM Trans. Graph. 34, 1, Article 11 (2014).

Beibei Liu, Yiying Tong, Fernando De Goes, and Mathieu Desbrun. 2016. Discrete Connection and Covariant Derivative for Vector Field Analysis and Design. ACM Trans. Graph. 35, 3, Article 23 (2016).

Scott A. Mitchell. 1996. A Characterization of the Quadrilateral Meshes of a Surface Which Admit a Compatible Hexahedral Mesh of the Enclosed Volume. Symp. Theor Comput. Sci. (1996), 465-476.

Ashish Myles, Nico Pietroni, and Denis Zorin. 2014. Robust Field-aligned Global Parametrization. ACM Trans. Graph. 33, 4, Article 135 (2014).

Ashish Myles and Denis Zorin. 2013. Controlled-distortion Constrained Global Parametrization. ACM Trans. Graph. 32, 4, Article 105 (2013).

Jonathan Palacios and Eugene Zhang. 2007. Rotational Symmetry Field Design on Surfaces. ACM Trans. Graph. 26, 3 (2007), Art. 55.

Daniele Panozzo, Enrico Puppo, Marco Tarini, and Olga Sorkine-Hornung. 2014. Frame Fields: Anisotropic and Non-orthogonal Cross Fields. ACM Trans. Graph. 33, 4, Article 134 (2014).

Nicolas Ray, Wan Chiu Li, Bruno Lévy, Alla Sheffer, and Pierre Alliez. 2006. Periodic Global Parameterization. ACM Trans. Graph. 25, 4 (2006), 1460-1485.

Jonathan R. Shewchuk. 1997. Adaptive Precision Floating-Point Arithmetic and Fast Robust Geometric Predicates. Discrete Comput. Geom. 18, 3 (1997), 305-363.

Kenshi Takayama, Daniele Panozzo, and Olga Sorkine-Hornung. 2014. Pattern-Based Quadrangulation for N-Sided Patches. Comput. Graph. Forum 33, 5 (2014), 177-184.

Amir Vaxman, Marcel Campen, Olga Diamanti, Daniele Panozzo, David Bommes, Klaus Hildebrandt, and Mirela Ben-Chen. 2016. Directional Field Synthesis, Design, and Processing. Comput. Graph. Forum 35, 2 (2016), 545-572.

Muyang Zhang, Jin Huang, Xinguo Liu, and Hujun Bao. 2010. A Wave-based Anisotropic Quadrangulation Method. ACM Trans. Graph. 29, 4, Article 118 (2010).

Afra J. Zomorodian. 2009. Topology for Computing. Cambridge University Press.

\section{A COMPATIBILITY OF QUADRANGULATIONS}

For seamless stitching of quadrangles between regular and singular regions, one may simply enforce a sufficient condition for compatibility as described below.

Proposition A.1. There exists a boundary-conforming quasi-dual MSC of a function $f$ on a mesh $\mathcal{D}$ when local maximum/minimum vertices of $\left.f\right|_{\partial \mathcal{D}}$ (vertices with smaller/greater values than the two adjacent boundary vertices) are also local maximum/minimum vertices of $\left.f\right|_{\mathcal{D}}$ (vertices with smaller/greater values than their one-ring neighbors).

Proof. Boundary extrema are nodes of the MSC just like any other extremum in $\mathcal{D}$. Moreover, each pair of consecutive minimum and maximum boundary vertices (as one travels along the boundary) belong to the same MSC cell-otherwise, a path connecting them would cross the border between MSC cells, which means the function values along the path have to increase and then decrease or decrease and then increase, but the boundary path between the two extremum boundary points is strictly monotonic. As any consecutive boundary extremum vertices belong to the same MSC cell, they are connected to a same saddle in MSC; thus they form one edge of the quasi-dual MSC cell around that saddle vertex. Consequently, $\mathcal{D}$ can be decomposed into quadrangular quasi-dual MSC cells conforming to the boundary.

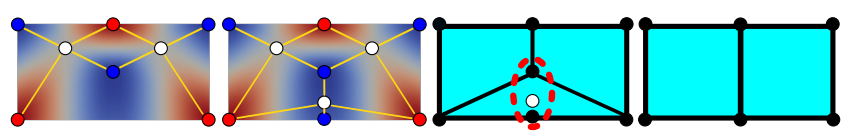

Fig. 19. Enforcing a proper quadrangulation. A Morse-Smale complex example (left) that does not quadrangulate the mesh (red/blue indicates high/low Morse function values resp.). This due to the boundary minimum at the bottom not being a minimum in the $2 \mathrm{D}$ domain. It can be easily fixed by perturbing the value at this boundary minimum. As the new saddle has a low Morse function value, the associated quadrangle can be removed by merging the two minimum vertices inside the red ellipse.

Note that the above condition implies that the number of quad edges connecting minimum and maximum boundary vertices is even, which is not true in general if the boundary is not derived from a Morse function, see Fig. 19.

Nevertheless, the construction with the $c c$ component as the Morse function makes the above condition satisfied: the boundary of the parameterized region contains boundary extremum points with values \pm 1 , which are already extremum points for the refined Morse-function inside the $\mathcal{M}_{S}$ patches. The boundary loops of these patches naturally have an even number of quadrangle edges to begin with, due to the enforcement of our transition condition $T_{1} \pm T_{2} \in 2 \mathbb{Z}$ when building the parameterization that leads to $Q_{R}$.

Due to limited floating point accuracy, an extremum on the boundary may not have its one-ring with values all above/below its own value as it should for Proposition A.1 to hold. We prevent this issue by turning values of extrema on boundary to $\pm(1+\epsilon)$ with a small positive $\epsilon$. With this treatment, seamless patching is guaranteed:

Proposition A.2. Given the cc component of $\Psi$ defined on $Q_{R}$ and a real function $f$ defined on a patch of $\mathcal{M}_{S}$ with matching boundary values $f(p)=c c(p) \forall p \in Q_{R} \cap \mathcal{M}_{S}$, there exists a quad mesh $Q_{S}$ defined by a perturbed $f$ such that $Q_{S}$ conforms to $Q_{R}$.

Proof. Any boundary extremum vertex of $f$ can be numerically turned into an extremum vertex of $\mathcal{M}_{S}$ by pushing it to a value slightly higher (resp., lower) than the maximum (resp., minimum) of its one-ring vertex values as discussed above. Then Proposition A.1. shows that there exists a quasi-dual MSC $Q_{S}$ with the same edges and vertices as $Q_{R}$ along the boundary of $\mathcal{M}_{S}$. 